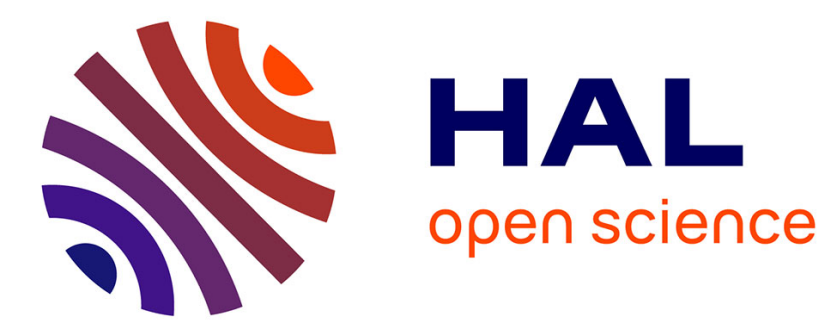

\title{
Sessions with an unbounded number of agents
}

Sundararaman Akshay, Loïc Hélouët, Madhavan Mukund

\section{To cite this version:}

Sundararaman Akshay, Loïc Hélouët, Madhavan Mukund. Sessions with an unbounded number of agents. 2014. hal-00979409v2

\section{HAL Id: hal-00979409 \\ https://hal.inria.fr/hal-00979409v2}

Preprint submitted on 23 Apr 2014

HAL is a multi-disciplinary open access archive for the deposit and dissemination of scientific research documents, whether they are published or not. The documents may come from teaching and research institutions in France or abroad, or from public or private research centers.
L'archive ouverte pluridisciplinaire HAL, est destinée au dépôt et à la diffusion de documents scientifiques de niveau recherche, publiés ou non, émanant des établissements d'enseignement et de recherche français ou étrangers, des laboratoires publics ou privés. 


\section{Sessions with an unbounded number of agents}

\author{
S. Akshay \\ IIT Bombay \\ Email: akshayss@cse.iitb.ac.in
}

\author{
Loïc Hélouët \\ INRIA Rennes \\ Email: loic.helouet@inria.fr
}

\author{
Madhavan Mukund \\ Chennai Mathematical Institute \\ Email:madhavan@cmi.ac.in
}

\begin{abstract}
In web-based business systems, agents engage in structured interactions, called sessions. Sessions are logical units of computations, like transactions. However, unlike transactions, sessions cannot be isolated from each other. Thus, one has to verify such systems in the presence of both intended and unintended interference between sessions.
\end{abstract}

The main challenge in building a tractable model of sessions is that there is no a priori bound on the number of concurrently active agents and sessions in the system. Realistic specifications require agents to compare entities across sessions, but this has to be modelled without assigning an unbounded set of unique identities to active agents and sessions.

We propose a model called session systems that allows for an arbitrary number of concurrently active agents and sessions. Agents are equipped with a limited ability to remember partners across sessions. Configurations are represented as graphs and the operational semantics is described through graph-rewriting. We show that, under reasonable restrictions, session systems are well-structured systems. This provides an effective verification algorithm for simple coverability properties. We then show how to use this result to verify more elaborate business rules such as avoidance of conflicts of interest and the Chinese Wall Property.

\section{INTRODUCTION}

Web services involve many parties interacting with each other to achieve a goal. The communication between the participating agents typically follows a structured protocol and the entire sequence of interactions can be seen as a logical unit of computation, typically called a session.

Sessions exhibit a richer behaviour than conventional transactions. Transactions combine smaller steps into computational units that satisfy the ACID properties-atomicity, consistency, isolation and durability. In particular, each transaction is assumed to be independent. When multiple transactions execute in parallel, the expected behaviour is specified in terms of notions such as serializability or linearizability that presuppose that transactions are atomic units that do not interact.

Sessions, on the other hand, typically need to interact to achieve the task at hand. Consider a scenario where a customer purchases an item from an online merchant and pays using a credit card. There are three interactions: the customer interacts with the merchant to order the item, the merchant interacts with the bank to confirm the payment and the customer interacts with the bank to authenticate the payment. Logically, each is a separate session. However, the merchant cannot confirm the order before the two sessions with the bank are completed. Likewise, the customer authenticates the payment after the merchant tells the bank how much is to be paid and before the bank confirms the payment to the merchant.
To capture these features, we need a model that does more than just encapsulating a sequence of activities as an atomic block. The model must permit controlled interactions such as the ones described above, while taking care to disallow undesirable interference. For instance, authentication for one payment should not be reused for another purchase.

Our goal is to build a tractable model of sessions that is amenable to formal verification. In addition to allowing sessions to interact in a controlled manner, there is another challenge. An unbounded number of agents of a given type may be active simultaneously - think of customers at an online store. This also allows unboundedly many sessions to be active in parallel. When sessions interact, we need to compare entities across sessions. If we naïvely use unique identifiers for agents and sessions, we have to deal with an unbounded set of names, which again makes verification intractable.

Our first contribution is to extend the session system formalism proposed in [1] to model systems with an arbitrary number of active agents and sessions. The original model only permitted a fixed and finite set of agents. We represent configurations of session systems as labelled graphs. The operational semantics is described in terms of finite graph rewriting rules.

Our second contribution is to propose effective verification techniques for session systems. Not surprisingly, reachability is undecidable for unbounded session systems. However, in many cases, the weaker property of coverability - whether a configuration embeds a given pattern-suffices. We show that, under reasonable restrictions, session systems fall within a class of well-structured transition systems (WSTS) for which coverability is decidable. We then use the decidability of coverability to verify properties expressing "business rules", such as avoidance of conflict of interest and the Chinese Wall Property (CWP) [2], which forbids an agent interacting with a company to have direct or indirect interactions at a later stage with a competitor. Our paper is organized as follows. Section II introduces session systems, and Section III defines their semantics. Section IV describes how to restrict session systems to well structured transition systems for which coverability is decidable. Section V assembles these results to provide effective verification tools to check conflicts of interest and CWPs. Several formalisms to describe or implement services and their orchestrations have been proposed in the past, and section VI lists some of them and compares with our approach and formalism. The complete operational semantics of session systems and proofs of theorems and important lemmas of the paper are provided in appendix. 


\section{SESSION SYSTEMS}

A session system represents the behaviours of (possibly infinite) sets of agents interacting with each other. Our model has two varieties of specifications. Agents behaviours are described by templates that determine how agents initiate and join sessions. Sessions are described by protocols that describe what happens during the course of a single structured interaction. We assume that any pair of actors in the system can communicate directly and reliably with each other whenever they need to. Hence, every actor of the system can share information with a partner as soon as it knows the identity of this partner. However, we make no further assumption about the way communication is implemented.

The key ingredient of our model is the session, a structured interaction among a finite set of agents to achieve a goal. An agent is an entity in the distributed system, e.g., a customer of an online store, a bank providing financial services online, ...

Agents operate at two levels. At an individual level, they can create, join and kill sessions, or query the system for the existence of a particular kind of session. At a collective level, they communicate with each other by playing different roles to define the interaction within sessions. Thus, an agent can participate in an unbounded number of sessions in parallel, and will be the creator (owner) of a subset of them.

Each agent manages a finite set of data variables that can be modified locally by the agent or during interactions within a session. In addition to data variables, each agent maintains a finite set of references to known agents. These references help in controlling interactions within a given scope.

Agents in the system follow predetermined behaviours, defined by templates called archetypes. Archetypes are transition systems with guards, whose moves are labelled either by agent operations to manage sessions (join, kill, create, query) or by assignments of variables. Figure 1 shows an example of archetype: an agent that is an instance of this (arche)type can create a session of type $S_{1}$, in which it must play the role 'client', then update variable $a$, join an arbitrary number of sessions of type $S_{2}$, and finally kill all sessions of type $S_{1}$ that it has created, provided the value of $a$ is true. We will explain later, in detail, the meaning of each of these transitions.

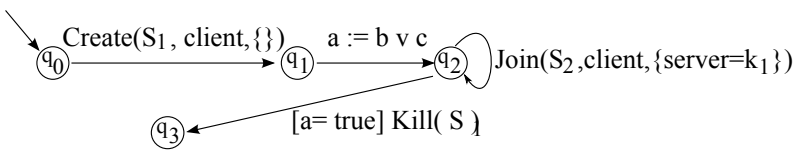

Fig. 1. An example of an archetype

As mentioned earlier, sessions are structured interactions involving a finite set of participating agents. Generic patterns of interactions are called protocols and are defined in terms of roles (e.g., client, merchant). Sessions are instances of these protocols in which roles are instantiated at runtime. We will say that a session $s$ is of type $P$ when $s$ is an instance of a protocol $P$. An agent can thus be involved in several sessions, and play different roles in each of them. For instance, an agent can be a client in one instance of a client-server protocol, and a server in another instance of the same protocol.
A protocol is a finite transition system labeled by guarded shared actions. An action is executed by a non-empty subset of roles participating in the protocol. This allows us to model not only synchronous actions or multicast communications (when an action is located on two or more agents), but also asynchronous message passing (when an action is executed by a single agent, and message sending and receptions are modelled by separate actions). In addition, an action involving a set of roles can assign values to the variables owned by agents playing these roles. This way, agents can exchange data values and agent identities.

Figure 2 shows an example of protocol. It represents an online sale with three roles, $\{C, S, D\}$, denoting, respectively, a customer, a store, and a delivery service. The shared actions are $\{$ Leave, Buy, ReadPage, Coordinates, BankInfo, CheckBalance, Cancel, Ship\}. The variables of the system include at least $\{\mathrm{Cl}$ ientAddress, myAddress, ClientBank, Mybank, Balanceok $\}$. The table summarizes how actions are shared. To simplify the example, we have abstracted the answer from the banking system through the action CheckBalance that non-deterministically sets the status of a client's account in the store. The meaning of this protocol is rather standard: a customer browses a website, then decides to buy, in which case he has to enter his personal information, and bank coordinates. The webstore then checks if the payment is granted by the customer's bank, in which case it asks for delivery of the chosen goods. Otherwise, it cancels the transaction. At any stage before payment, the customer can leave the transaction.

A session may start as soon as it is created, even if all its roles are not yet assigned. Consider, for instance, a protocol modelling a chat service with three roles, one server and two clients. A chat session can be established as soon as one client and the server are ready. The second user may join an existing session later. However, since we allow shared actions among sets of roles, a shared action can occur only when all roles that participate in it have been assigned to agents.

A session system manages an arbitrary number of parallel sessions with an unbounded number of client requests. Handling multiple sessions at the same time raises security issues, and one has to take side effects into account when performing an action within a session. For instance, the example of Fig-

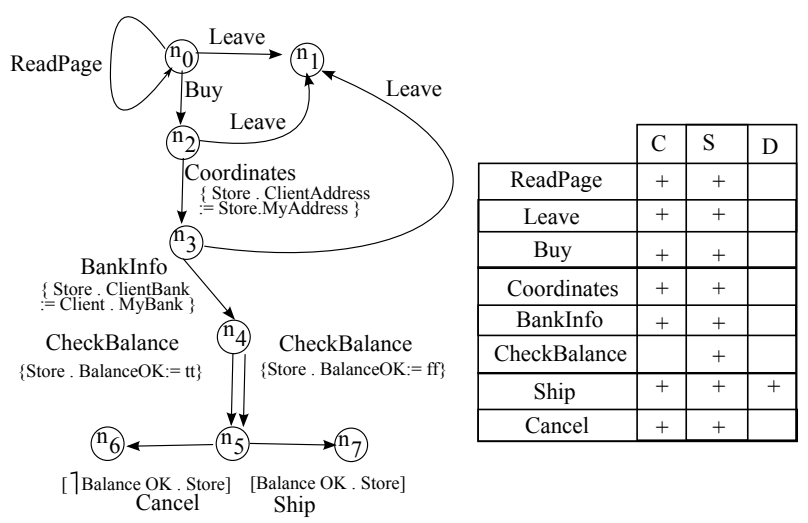

Fig. 2. An example of a protocol 
ure 2 may seem correct when considering one single session, or when considering that sessions are handled one after the other. However, if several sessions coexist in the system, the balance status should not be stored in a global variable by the online merchant. Identifying such problems calls for automated verification tools, which is the focus of this paper.

We now formalize the notions of archetypes and protocols. As mentioned earlier, each agent maintains a finite set of data variables, assumed to range over finite domains. For simplicity, we consider only boolean variables, since finite domains can be encoded using combinations of boolean values. We assume that all agents have the same set of (boolean) data variables and denote this set by $\mathcal{V}$. We assume that $\mathcal{V}$ contains a special variable blocked, to encode the status of an agent. In addition, each agent has a finite set of reference variables, or just references, that point to other agents in the system. As with data variables, we assume that all agents have the same set of reference variables, denoted $\mathcal{K}$. The set of Boolean expressions over $\mathcal{V} \cup \mathcal{K}$ is defined as follows:

$$
\begin{aligned}
\phi::= & \text { true } \mid \text { false }|v| \neg v\left|\phi_{1} \wedge \phi_{2}\right| \phi_{1} \vee \phi_{2} \mid \\
& k_{1}=k_{2}\left|k_{1} \neq k_{2}\right| \phi_{1}=\phi_{2} \mid \phi_{1} \neq \phi_{2},
\end{aligned}
$$

where $v \in \mathcal{V}$, and $k_{1}, k_{2} \in \mathcal{K}$, and $\phi_{1}, \phi_{2}$ are expressions. Note that negations have been pushed down to atomic formulas and only conjunctions and disjunctions are allowed at the top level.

Variables and references can be updated through assignments. Assignments are denoted by $b:=e$ where $b \in \mathcal{V}$ and $e$ is a boolean expression over $\mathcal{V} \cup \mathcal{K}$ (boolean assignment), or by $k:=k^{\prime}$ where $k, k^{\prime} \in \mathcal{K}$ (reference assignment). In general, given a set of variables $\mathcal{X}$, we write $\operatorname{Expr}(\mathcal{X})$ for the set of boolean expressions over $\mathcal{X}$ and $A s g(\mathcal{X})$ for the set of valid assignments to $\mathcal{X}$.

As usual, a valuation for a set of variables $\mathcal{X}$ is a function $\operatorname{val}()$ that maps each variable in $\mathcal{X}$ to an element of its domain. Given a valuation $\operatorname{val}()$ and an expression $e \in \operatorname{Expr}(\mathcal{X})$, we write $\operatorname{val}()=e$ if the expression $e$ evaluates to true under the valuation $\operatorname{val}()$. We say that expression $e$ is satisfiable if there exists such a valuation for $\mathcal{X}$.

We are now ready to formally define session systems. We define $\Sigma$, the set of agent operations, as follows.

$$
\begin{aligned}
\Sigma=a \mid & \operatorname{Spawn}(s, r, c)|\operatorname{Kill}(s)| \operatorname{Join}(s, r, c) \mid \\
& \operatorname{Bjoin}(s, r, c) \mid Q \operatorname{Query}(s, c),
\end{aligned}
$$

where $a \in A s g(\mathcal{V} \cup \mathcal{K}), s$ is the name of a protocol, $r$ is a role in $s$ that the agent intends to play and $c$ is a constraint on the identity of other agents within this protocol. Constraints are boolean combinations of atoms of the form $r_{p}=r_{q} \cdot k_{j}$ in which $r_{p}, r_{q}$ are roles of protocol $s$ and $k_{j} \in \mathcal{K}$. Such a constraint expresses the fact that role $r_{p}$ can only be played by the agent that is referred to as $k_{j}$ by the agent playing role $r_{q}$ in a protocol. With this mechanism, an agent can ask to join a session with a particular agent known to it, or, conversely, forbid undesirable agents that it knows about from participating in sessions it creates. We denote by $\operatorname{Cnst}(s, \mathcal{K})$ the finite set of possible constraints on roles of a protocol $s$ using variables $\mathcal{K}$.

We now explain the agent operations in $\Sigma$. $\operatorname{Spawn}(s, r, c)$ creates a session of type $s$ in which the agent creating the session plays role $r$, and the remaining roles can only be assigned to agents satisfying the constraint $c \in \operatorname{Cnst}(s, \mathcal{K})$. $\operatorname{Join}(s, r, c)$ asks to join a session of type $s$, where the requesting agent wishes to play role $r$. This join request is kept in a set of pending requests and the agent can proceed to perform other operations. $\operatorname{Bjoin}(s, r, c)$ is similar to $\operatorname{Join}(s, r, c)$, but blocks the requesting agent until an existing session of the desired type is joined. Query $(s, c)$ asks if there is a current session of type $s$ satisfying constraint $c$ and blocks the agent so long as there exists no such session. When an agent is blocked, its blocked variable is set to $t t$. $\operatorname{Kill}(s)$ kills all sessions of type $s$ owned by the executing agent in the system.

Definition 1: An archetype is a tuple of the form $\left(Q, q_{0}, \Delta\right)$, where:

- $Q$ is a finite set of states, with $q_{0}$ the initial state,

- $\Delta \subseteq Q \times \operatorname{Expr}(\mathcal{V} \cup \mathcal{K}) \times \Sigma \times Q$ is a transition relation, where $\Sigma$ is the set of agent operations.

A transition in $\Delta$ is a tuple $\left(q, g, \sigma, q^{\prime}\right)$, where $q, q^{\prime}$ are states of the archetype, $g \in \operatorname{Expr}(\mathcal{V} \cup \mathcal{K})$ is a guard, and $\sigma \in \Sigma$ an agent operation. Archetypes describe how sessions are handled at a high-level by agents. An agent can move from one state to another via a transition and perform the associated agent operation provided the guard of the transition holds in the current valuation. The complete operational semantics of Spawn, Join, Bjoin, Query and Kill is given in appendix.

Next, we define templates for structured interactions between agents, called protocols. A protocol $S$ is an automaton whose transitions are labeled by shared actions. The participants in an action are a subset of the roles $\mathcal{R}$ associated with the protocol. We write $r . v$ to refer to the variable $v$ attached to role $r \in \mathcal{R}$. Hence, protocols are transition systems whose guards and assignments are defined over $\mathcal{R} . \mathcal{V}=\{r . v \mid v \in$ $\mathcal{V}, r \in \mathcal{R}\}$ and $\mathcal{R} . \mathcal{K}=\{r . k \mid k \in \mathcal{K}, r \in \mathcal{R}\}$. where

Definition 2: A protocol is a tuple $S=\left(N, n_{0}, \delta, \mathcal{R}, \Gamma, l\right)$,

- $\quad N$ is a finite set of session nodes, $n_{0}$ is an initial node,

- $\Gamma$ is a finite alphabet of actions,

- $\mathcal{R}=\left\{r_{1}, \ldots r_{n}\right\}$ is a finite set of roles,

- $l: \Gamma \times \mathcal{R} \rightarrow\{\perp,+, \top\}$ is a function that indicates whether role $r$ participates in the shared action $\gamma$ and whether this action is the last one performed by this agent in the current session. More precisely, $l(\gamma, r)=$ $\perp$ if role $r$ does not participate in $\gamma, l(\gamma, r)=\top$ if role $r$ participates in $\gamma$ and $\gamma$ is its last action in the current session, and $l(\gamma, r)=+$ if role $r$ participates in $\gamma$ but $\gamma$ is not its last action in the current session.

- $\delta \subseteq N \times \operatorname{Expr}(\mathcal{R} . \mathcal{V} \cup \mathcal{R} . \mathcal{K}) \times \Gamma \times 2^{A s g(\mathcal{R} . \mathcal{V} \cup \mathcal{R} . \mathcal{K})} \times N$ is a transition relation.

Sessions are instances of protocols with concrete agents assigned to roles. As all roles need not be defined simultaneously, a session may start with some roles still unassigned. A transition $\left(n, g, \gamma, a s, n^{\prime}\right)$ in the protocol indicates that the session can move from node $n$ to $n^{\prime}$ through the shared action $\gamma$ provided guard $g$ holds with respect to the current values of $\mathcal{R} . \mathcal{V} \cup \mathcal{R} . \mathcal{K}$ and all roles involved in $\gamma$ (i.e., $r$ such that $l(\gamma, r) \in\{+, \top\})$ have been assigned to agents. Executing $\gamma$ results in the update of variables: the variables 
$R . \mathcal{V} \cup R . \mathcal{K}$ are modified as described in the set of assignments as. Note that a shared action allows multiple assignments, performed atomically, in parallel. Practically, performing actions involving more than one agent would require use of a shared memory, or synchronization among participants of the session. To ease up implementation, one could also require actions to be local to a single agent, and communications to be asynchronous. However, these implementation details do not affect the decidability results described in this paper, and are left for future work.

Definition 3: A session system is a pair $S S=(\mathcal{A}, \mathcal{S})$ where $\mathcal{A}=\left\{A_{1}, \ldots, A_{k}\right\}$ is a finite set of archetypes and $\mathcal{S}=\left\{S_{1}, \ldots, S_{q}\right\}$ is a finite set of protocols. Each archetype in $\mathcal{A}$ is of the form $A_{i}=\left(Q_{i}, q_{0}^{i}, \Delta_{i}\right)$, and each protocol in $\mathcal{S}$ is of the form $S_{j}=\left(N_{j}, n_{0}^{j}, \delta_{j}, \mathcal{R}_{j}, \Gamma_{j}, l_{j}\right)$

\section{SESSION SYSTEMS SEMANTICS}

In this section, we describe the operational semantics of session systems. A configuration of a session system describes the local states of all agents and sessions, together with the valuation of their variables. We represent a configuration as a (vertex and edge) labeled graph $C=(V, E, \tau)$ where

- $V=V_{A} \uplus V_{S}$ is a finite set of vertices, where $V_{A}$ denotes agents (i.e., instanciations of archetypes) and $V_{S}$ denotes sessions (i.e., instanciations of protocols).

- $E$ is a set of labeled edges over $V$, representing agent references, and connections between agents and sessions. We have $E \subseteq\left(V_{A} \times \mathcal{K} \times V_{A}\right) \cup\left(V_{A} \times\right.$ $\left.\mathbb{N} \times\{\mathrm{tt}, \mathrm{ff}\} \times V_{S}\right) \cup\left(V_{A} \times\{q r y\} \times V_{S}\right)$. A triple $\left(v, k, v^{\prime}\right) \in V_{A} \times \mathcal{K} \times V_{A}$ represents the fact that the agent $A$ represented by vertex $v$ has the identity of agent $A^{\prime}$ represented by vertex $v^{\prime}$ stored via reference variable $k$. An edge of the form $\left(v, q r y, v^{\prime}\right)$ means that the agent represented by vertex $v$ is querying the system for a specific kind of session, whose characteristics are described by $v^{\prime}$. The number of labels for these two kinds of edges is of course finite. Edges of the form $(v, l, s)$ where $l=(n, b) \in \mathbb{N} \times\{\mathrm{tt}$, ff $\}$, $v \in V_{A}$, and $s \in V_{S}$ indicates one of the following.

$\circ \quad$ The agent represented by vertex $v$ plays role $n$ in a session represented by vertex $s$. The boolean flag $b$ is set to tt if $v$ is the owner of the session, and to false otherwise.

- The agent represented by vertex $v$ asks to play role $n$ in a session. Vertex $s$ then represents a join request. The label of this vertex defines the type of session agent that $v$ wishes to join, along with the constraints attached to this request. Here, the flag $b$ is set to $\mathrm{ff}$ ( $v$ cannot own of a joined session that it did not create).

Note that an agent can be connected via reference edges only to a finite number of agents, but it can be connected to an unbounded number of session vertices. Conversely, a session can only be connected to a finite number of agents, that is at most the number of roles in the protocol it instanciates.

- $\quad \tau$ is a labeling of each vertex of $V_{A}$ with details of the agent it represents (archetype, control state, valuation of variables) and each vertex of $V_{S}$ with details of the session it represents (protocol name, current node, constraints on roles yet to be instantiated).

More formally, the labelling of vertices is defined as follows. We have $\tau: V_{A} \rightarrow \mathcal{A} \times \bigcup Q_{i} \times \operatorname{Val}(\mathcal{V})$. $i \in \mathcal{A}$

The labelling $\tau(v)=\left(A_{i}, q, v a l\right)$ indicates that $v$ represents an agent behaving according to archetype $A_{i}$, currently in state $q \in Q_{i}$, with valuation val of its variables. Let us now consider labeling of session vertices. We have $\tau: V_{S} \rightarrow \mathcal{S} \times \mathcal{N} \times \operatorname{Cnst}(\mathcal{S}, \mathcal{K}) \times 2^{\mathcal{R}}$ where $\mathcal{N}$ is the union of all possible nodes appearing in protocols. For every vertex $v \in V_{S}$ we have $\tau(v)=(s, n, c, J)$ if $v$ represents an instance of a running session. A tuple $(s, n, c, J)$ indicates that vertex $v$ represents an instance of protocol $s$, currently in node $n$ with contraints $c$ attached to uninstantiated roles, such that roles in $J$ have not yet been assigned. Similarly, we have $\tau(v)=(s, c)$ if $v$ represents a join request or a query for a protocol $s$ with constraints defined by $c$. In such case, vertex $v$ is connected to an agent vertex either via an edge labeled by $(r, \mathrm{ff})$ for a join request, or via an edge labeled by qry for a query.

Clearly, the set of labels attached to edges and vertices is finite. We further impose well-formedness constraints. For every vertex $v \in V_{S}$, if $\tau(v)=(s, c)$ then there exists a single vertex $v \in V_{A}$ connected to $v$. If $\tau(v)=(s, n, c)$, then the number of agents connected to $v$ is at most the number of roles in protocol $s$. Further, exactly one agent is connected to a session via an edge labeled by $(n, \mathrm{tt})$, i.e., each session has only one owner.

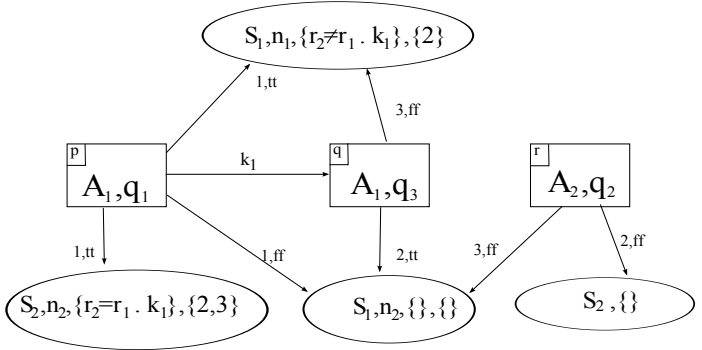

Fig. 3. Graphical representation of configurations

Figure 3 shows a graphical representation of a possible configurations for a set of archetypes $\mathcal{A}=\left\{A_{1}, A_{2}\right\}$ and a set of protocols $\mathcal{S}=\left\{S_{1}, S_{2}\right\}$. Agents are represented as rectangles and sessions as elipses. For clarity, we have not represented variable valuations in the labels of agents. In this configuration, an agent $p$ of (arche)type $A_{1}$ is in state $q_{1}$, another agent $q$ of type $A_{1}$ is in state $q_{3}$, and a third agent $r$ of type $A_{2}$ is in state $q_{2}$. Agent $p$ knows agent $q$, and has stored its address in its reference variable $k_{1}$. Agents $p, q$ and $r$ are involved in a session of type $S_{1}$ owned by $q$, currently in state $n_{2}$. Agents $p, q, r$ play roles $r_{1}, r_{2}, r_{3}$, respectively, within this instance of $S_{1}$. Agent $p$ is the owner and plays role $r_{1}$ in an instance of session protocol $S_{2}$, currently in node $n_{2}$. A constraint indicates that role $r_{2}$ can only be assigned to the agent known as $k_{1}$ by $r$, and roles $r_{2}, r_{3}$ are not yet assigned. Agents $p, q$ have joined a session of type $S_{1}$ and play roles $r_{1}$ and $r_{3}$, respectively, within the session. Agent $p$ is the owner of this session, and the constraint says that the agent that will join as role $r_{2}$ should differ from the agent known by $p$ as 
entry $k_{1}$ in its set of references. Agent $r$ has asked to join an existing session of type $S_{2}$, as role $r_{2}$.

In the rest of the paper, we denote by $\mathcal{C}$ the (possibly infinite) set of all configurations. We provide an informal semantics for our session systems (a detailed semantics is available in appendix). A session system starts in an initial configuration $C_{0}$ (typically the empty configuration). Three kinds of moves can occur from a configuration: a local move of an agent, a shared action within a session, or an environment move, i.e., either an operation that matches a join request to an existing session or existing query, or the arrival of a new agent in the system. Local agent moves can change the values of the variables owned by the agent, create a new session, ask to join a particular kind of session, kill sessions owned by the agent, or modify its references. All these actions are described in our model through a finite number of creations or deletions of vertices and edges, and a relabeling of vertices, due to change of valuation and state of the agent. Spawn creates a new session vertex and connects it to the creating agent. Each form of join (Join, Bjoin) and query also creates a new vertex and a new edge with appropriate labels corresponding to the operation. Kill suppresses edges and session vertices corresponding to some sessions owned by the agent. As all these operations are transitions of an archetype, this also results in a relabeling of the agent vertex to model the change of state. Session actions model interactions among agents within a session: in some currently active session, a shared action that is currently enabled is performed. Such a move results in a relabeling of a session vertex in a configuration (the session changes its state), a relabeling of agents involved in the session (the agents variable may be updated), and a finite number of changes in the edges representing the references of agents contributing to the session.

Environment moves are high-level actions provided by a system that manages all sessions. We do not detail how such system is implemented. Arrival of a new agent introduces a fresh instance of some archetype into the system (it creates a new agent vertex). Agent vertices are never removed. Query unlocking simply consists in removing the edge and vertex modeling a query, and changing the querying agent's label to model the change in status of the special variable blocked. The last kind of move is servicing a join request. This operation nondeterministically matches a pending join request and an available session. It results in the suppression of the vertex representing the request, a connection of the joining agent to a session vertex with an appropriate label, and in a relabeling of the vertex representing the joined session to take into account the constraints imposed by the joining agent. Note that join requests can remain pending for an arbitrarily long time, even if matching sessions are available. There is no obligation to match pending join requests "eagerly".

Following this informal definition of session systems semantics, we can define the behaviours of a session system as sequences of moves satisfying the semantics. A move from a configuration $C$ to a configuration $C^{\prime}$ via action $\sigma$ is denoted by $C \stackrel{\sigma}{\longrightarrow} C^{\prime}$. A run of a session system from a configuration $C_{0}$ is a sequence of moves $\rho=C_{0} \stackrel{\sigma_{1}}{\longrightarrow} C_{1} \ldots \stackrel{\sigma_{k}}{\longrightarrow} C_{k}$. Given a set of configurations $X$, we will say that $\rho$ is a run over $X$ if it is exclusively composed of configurations from $X$. With this semantics, session systems define infinite state transition systems. We refer interested readers to the formal semantics of moves defined in appendix. We will say that a configuration $C$ is reachable from an initial configuration $C_{0}$, denoted $C_{0} \longrightarrow C_{k}$, if there exists $\rho=C_{0} \stackrel{\sigma_{1}}{\longrightarrow} C_{1} \ldots \stackrel{\sigma_{n}}{\longrightarrow} C$.

Theorem 4: Reachability of a configuration $C$ from an initial configuration $C_{0}$ is undecidable.

A similar result was proved in [1] for a less expressive session model. Session systems can simulate reset Petri nets, for which reachability is undecidable. Sessions can be used to encode place contents, adding tokens is simulated by creating sessions, consuming them is simulated by entering a session and terminating it, and resetting places is simulated by killing sessions corresponding to the reset places.

\section{Well-STRUCTURED SESSION SyStems}

As observed above, session systems have an infinite configuration space and as a result verifying even simple reachability properties is undecidable. However, we will now show that under some mild restrictions, these systems are well-structured, which implies that some interesting problems (such as checking coverability) are decidable. In the next section, we will show that this allows to verify interesting properties and in particular business rules such as conflict of interest.

We start with some relevant notions and results from [3]. A well-quasi ordering (WQO) on a set $X$ is a reflexive, transitive binary relation $\leq$ such that any infinite sequence $x_{0}, x_{1}, \ldots$ of elements of $X$ contains a pair $x_{i}, x_{j}$ such that $i<j$ and $x_{i} \leq$ $x_{j}$. In fact, $\leq$ is a WQO iff it is well-founded on $X$ (i.e. it does not contain infinite decreasing sequences) and does not contain infinite antichains, i.e., infinite sets of incomparable elements. The upward closure of a set $X$ is $\uparrow X=\{y \mid \exists x \in X, y \geq x\}$. A set $X$ is called upward closed set if $\uparrow X=X$. An upward closed set $X$ in a WQO can be represented by a finite basis $B(X)=\min _{\leq}\{X\}$. This property is particularly useful: as $X=\cup_{x \in B(X)} \uparrow\{x\}$, one can recall infinite upward closed sets of elements using only a basis as finite set of representatives.

A well-structured transition system (WSTS) is a structure $(X, s u c c, \leq)$ where $X$ is a possibly infinite set of elements, succ $\subseteq X \times X$ is a transition relation, and $\leq$ is a preorder on $X$ such that $(X, \leq)$ is a wqo, and succ satisfies the following monotonicity property: $\forall x \in X,\left(x, x^{\prime}\right) \in$ succ and $x_{1} \geq$ $x$ implies $\left(x_{1}, x_{1}^{\prime}\right) \in$ succ $^{*}$ for some $x_{1}^{\prime} \geq x^{\prime}$, where succ* is the transitive and reflexive closure of relation succ. For an upward closed set $X$, we denote by $\operatorname{pre}(X)=\{y \mid \exists x \in X, x \in$ $\operatorname{succ}(y)\}$ the set of predecessors of $X$ (by relation succ). $\operatorname{pre}^{*}(X)=\left\{y \mid \exists x \in X, x \in \operatorname{succ}^{*}(y)\right\}$. The pred-basis of $X$ is the finite set $\operatorname{pred}_{B}(X)=B($ pre $(X))$. We say that a WSTS has effective pred-basis if there exists an algorithm that accepts an element $x$ and returns a basis for $\uparrow(\operatorname{Pre}(\uparrow x))$. We recall the following result (see for instance [3]):

Proposition 5 (Coverability in WSTS [3]): Let $S=(X, \leq, s u c c)$ be a WSTS with decidable $\leq$ and effective pred basis, then for a pair $x, x_{0} \in X$, one can decide the coverability problem, which asks whether there exists a run of $S$ from $x_{0}$ to some $x^{\prime}$ such that $x^{\prime} \geq x$.

Let us lift the result of proposition 5 to sessions systems. For this, we define an ordering on configurations. 
Definition 6: Let $C_{1}=\left(V_{1}, E_{1}, \tau_{1}\right), C_{2}=\left(V_{2}, E_{2}, \tau_{2}\right)$ be two configurations of a session system. We will say that $C_{1}$ is a subgraph of $C_{2}$, denoted by $C_{1} \sqsubseteq C_{2}$ iff there exists a pair of mappings $\psi: V_{1} \rightarrow V_{2}$ and $\psi^{\prime}: E_{1} \rightarrow E_{2}$ such that:

- $\forall v \in V_{1}, \tau(\psi(v))=\tau(v)$

- $\quad \forall e=\left(v, l, v^{\prime}\right) \in E_{1}$, with $l \in \mathcal{K} \cup(\mathbb{N} \times\{t t, f f\}) \cup$ $\{q r y\}$, then $\psi^{\prime}(e)=(\psi(v), l, \psi(v))$ is an edge of $E_{2}$.

We will also say that two configurations $C_{1}, C_{2} \in \mathcal{C}$ are isomorphic iff $C_{1} \sqsubseteq C_{2}$ and $C_{2} \sqsubseteq C_{1}$. As configurations are finite graphs, one can effectively check if $C_{1} \sqsubseteq C_{2}$.

Now if $(\mathcal{C}, \longrightarrow, \sqsubseteq)$ were a WSTS, we could directly check properties of session systems using Proposition 5 above. However, $(\mathcal{C}, \sqsubseteq)$ is not a WQO: one can design sets of pairwise incomparable configurations of arbitrary sizes. To overcome this problem, we need to restrict the set of configurations considered. One obvious restriction is obtained by setting a bound on the number of agents in configurations. In [1], we have shown that this suffices to obtain a WQO on configurations (roughly speaking, the set of configurations can be encoded as an integer vector counting the number of occurrences of each tuple (session, state, unassigned roles $\rangle$ ). However, for some applications, this can be rather restrictive. For instance, a webstore is supposed to accept huge numbers of clients. Even if its resources call for a bound on the number of clients using a site, this bound depends on architectural choices, and not on the behavioural specification of the system (increasing the resource may increase the number of clients a store can handle), and one can not set such bound a priori. In this paper, we propose a different restriction, namely $k$ boundedness of configurations, which allows us to model systems with unboundedly many agents and sessions, and yet obtain a WQO on configurations.

Let $C=(V, E, \tau)$ be a configuration. We will say that a vertex $v_{2} \in V_{A}$ is a successor of vertex $v_{1} \in V_{A}$ iff there exists a reference edge from $v_{1}$ to $v_{2}$ (i.e, $\left(v_{1}, k_{i}, v_{2}\right) \in E$ for some $\left.k_{i} \in \mathcal{K}\right)$ or if there exists a pair of edges $\left(v_{1}, l, s\right),\left(v_{2}, l^{\prime}, s\right) \in$ $E$ connecting the two agent vertices to the same session vertex $s$. A path of $C$ is a sequence of vertices $v_{1}, v_{2} \ldots v_{n}$ such that $v_{i+1}$ is a successor of $v_{i}$ in $C$. This path is simple if $v_{i} \neq v_{j}$ for every $i \neq j$, and the length of a simple path is its number of vertices. A configuration $C$ is $k$-bounded if it has no simple paths of length greater than $k$.

In the rest of the paper, we will denote by $\mathcal{C}$ the set of all configurations, and by $\mathcal{C}^{k}$ the set of $k$-bounded configurations. Note that $\mathcal{C}^{k}$ is not a finite set: configurations of $\mathcal{C}^{k}$ may contain an arbitrary number of $k$-bounded connected components, containing arbitrary numbers of sessions. So, the number of vertices in configuration of $\mathcal{C}^{k}$ is not bounded, but the way these vertices are connected is constrained.

The only way for a configuration $C \in \mathcal{C}^{k}$ to evolve into $C^{\prime} \notin \mathcal{C}^{k}$ is through a merge move by the environment, or by learning the address of a new agent, which creates a new simple path of length greater than $k$. In such a situation, that is, when $C \stackrel{\sigma}{\longrightarrow} C^{\prime}$ with $C \in \mathcal{C}^{k}$ and $C^{\prime} \in \mathcal{C}^{k^{\prime}>k}$ for some action $\sigma$, we consider that the system leaves the set of accepted configurations, and we replace this move by a move $C \rightarrow \top$ to a special configuration $T$ ("Top") that has the following properties:
- $C \sqsubseteq \top$ for all configurations $C \in \mathcal{C}^{k} \cup\{\top\}$. Henceforth we write $\mathcal{C}_{\top}^{k}$ for $\mathcal{C}^{k} \cup\{\top\}$.

- All types of moves (session, agent, environment) are enabled at $T$ and the resulting configuration is $T$.

The element $T$ should not be considered as a real configuration, but rather as an indication that a run of the system has reached a configuration that is not $k$-bounded. We are now ready to prove that this restriction to $k$-bounded configurations provides the well-structured property.

Theorem 7: For a fixed $k \in \mathbb{N},\left(\mathcal{C}_{\top}^{k}, \longrightarrow, \sqsubseteq\right)$ is a wellstructured transition system.

Proof sketch: We first show that $\left(\mathcal{C}_{\top}^{k}, \sqsubseteq\right)$ is a WQO, by adapting the result of [4], which shows that labelled undirected graphs of $k$-bounded path length, ordered by the induced subgraph relation is a WQO. Then, the transition relation $\longrightarrow$ is compatible with $\sqsubseteq$, as guards are monotonous w.r.t. $\sqsubseteq$, and as all transitions are finite sequences of graph transformations preserving $\sqsubseteq$.

We have proved that $\left(\mathcal{C}_{T}^{k}, \longrightarrow, \sqsubseteq\right)$ is a WSTS, and, by definition, $\sqsubseteq$ is effective. But in order to use Proposition 5 to show decidability of coverability for session system, we still need to prove that computation of a pred basis is effective, which we shall do now.

In the rest of the paper, we will use set saturation techniques, and represent upward closed sets as their basis. Indeed, as $\mathcal{C}_{T}^{k}$ is a WQO, any upward closed set of configurations has a finite basis: for a configuration $C$ in $\mathcal{C}_{T}^{k},\{C\}$ is a finite basis for $\uparrow C$. This property extends to arbitrary sets of configurations. For any finite $\left\{C_{1}, C_{2}, \ldots, C_{n}\right\} \subseteq \mathcal{C}_{\top}^{k}$, the basis of $\bigcup_{i=1}^{n} \uparrow C_{i}$ is the set of minimal elements w.r.t. $\sqsubseteq$ in $\left\{C_{1}, C_{2}, \ldots, C_{n}\right\}$. Using bases as representations for upward closed sets in a WQO setting allows us to work with finite representations of infinite sets. Further, it is sufficient to manipulate a basis $B=\left\{B_{1}, B_{2}, \ldots, B_{n}\right\}$ to decide membership. If $X$ is an upward closed subset of $\mathcal{C}^{k}$ represented by its finite basis $\left\{B_{1}, B_{2}, \ldots, B_{n}\right\}$, then checking that $C \in X$ amounts to checking $B_{i} \sqsubseteq C$ for some $B_{i}$ in the basis of $X$.

The usual definitions of Pre and upward closure apply to $\left(\mathcal{C}_{\top}^{k}, \longrightarrow, \sqsubseteq\right)$. For any configuration $C \in \mathcal{C}_{\top}^{k}$, we define $\operatorname{Pre}(C)=\left\{C^{\prime} \mid C^{\prime} \stackrel{\sigma}{\rightarrow} C\right\}$. The relation extends to upward closures as follows: $\operatorname{Pre}(\uparrow C)=\left\{C^{\prime} \mid C^{\prime} \stackrel{\sigma}{\rightarrow} C^{\prime \prime} \sqsupseteq C\right\}$, and to sets of configurations as follows: for $X \subseteq \mathcal{C}_{\top}^{k}$, $\operatorname{Pre}(X)=\bigcup_{C \in X} \operatorname{Pre}(C)$ and $\operatorname{Pre}(\uparrow X)=\bigcup_{C \in X} \operatorname{Pre}(\uparrow C)$. Computing a basis for an upward closed set is easy: a basis is defined as the minimal elements of a set w.r.t. $\sqsubseteq$, which guarantees that computing this basis is effective. Computing a pred-basis is also effective, and amount to computing the finite set of predecessors of elements in a basis, and then the minimal elements of this set. We then have the following lemma:

Lemma 8: Let $X$ be an upward closed subset of $\mathcal{C}_{\text {T}}^{k}$ represented by it basis $\left\{B_{1}, B_{2}, \ldots, B_{n}\right\}$. Then we can effectively compute a basis for $\uparrow \operatorname{Pre}(X)$.

In the context of session systems, the coverability problem consists of deciding if, from an initial configuration $C_{0}$, one can reach a configuration $C^{\prime}$ that covers a target configuration $C$ (i.e., such that $C \sqsubseteq C^{\prime}$ ). When $C$ is coverable from $C_{0}$, we write $C_{0} \leadsto C^{\prime}$. Similarly, we will say that a run $\rho=C_{0} \stackrel{\sigma_{1}}{\longrightarrow}$ 
$C_{1} \ldots \stackrel{\sigma_{k}}{\longrightarrow} C_{k}$ covers $C$ if $C \sqsubseteq C_{k}$. The properties of WSTSs, as well as the results of Theorem 7 and Lemma 8 allow us to state the following corollary on decidability of coverability in session systems:

Corollary 9: For a sessions system $S=(\mathcal{A}, \mathcal{S})$, coverability of a configuration $C$ by a run over $\mathcal{C}^{k}$ from an initial configuration $C_{0}$ is decidable.

Proof: We show that there is an effective algorithm to check, for a given configuration $C$ and a session system $S$, if there exists a run of $S$ starting from a configuration $C_{0}$ that reaches a configuration that subsumes $C$.

We start from $C$. We know that $\{C\}$ is a basis for $\uparrow C$. Furthermore, any run that ends in a configuration in $\uparrow C$ is a run that covers $C$. We are hence looking for a run that starts from $C_{0}$ and reaches a configuration in $\uparrow C$. Such a run exists iff $C_{0} \in \uparrow p r e^{*}(\uparrow C)$, or equivalently, if $C_{b} \sqsubseteq C_{0}$ for some $C_{b} \in \operatorname{Basis}\left(\uparrow p r e^{*}(\uparrow C)\right)$. As $\left(\mathcal{C}_{\top}^{k}, \longrightarrow, \sqsubseteq\right)$ is a WSTS (by Theorem 7) with effective $\sqsubseteq$ and effective pred basis computation (by Lemma 8), one can use a set-saturation algorithm to compute a basis for $\uparrow p r e^{*}(\uparrow C)$. The algorithm to compute a basis $P B=\operatorname{Basis}\left(\uparrow p r e^{*}(\uparrow C)\right)$ is a fixpoint algorithm. We start from $P B_{0}=\operatorname{Basis}(\uparrow C)=\{C\}$, and then compute iteratively $P B^{k}=P B^{k-1} \cup \operatorname{Basis}\left(\uparrow \operatorname{pre}\left(\uparrow P B^{k-1}\right)\right)$, and stop as soon as $\uparrow P B^{k}=\uparrow P B^{k-1}$. It has been proved in [3] that for any upward closed set $X$, this algorithm is correct and terminates when the pred-basis computation is effective. Hence, at the end of the fixpoint computation, we have $\uparrow \cup P B^{k}=\operatorname{Pre} e^{*}(\uparrow C)$.

Now, for any element $C_{b}$ in $P B$, there exists a run $\rho_{b}$ from $C_{b}$ to a configuration $C_{b}^{\prime}$ that covers $C$. Hence, if $C_{b} \sqsubseteq C_{0}$ there exists a run over the same actions than $\rho_{b}$ from $C_{0}$ to a configuration greater than $C_{b}^{\prime}$, and hence greater than $C$. As $\sqsubseteq$ is effective, it then suffices to build the basis $P B$, and compare its elements to $C_{0}$ to check if $C$ is coverable from $C_{0}$.

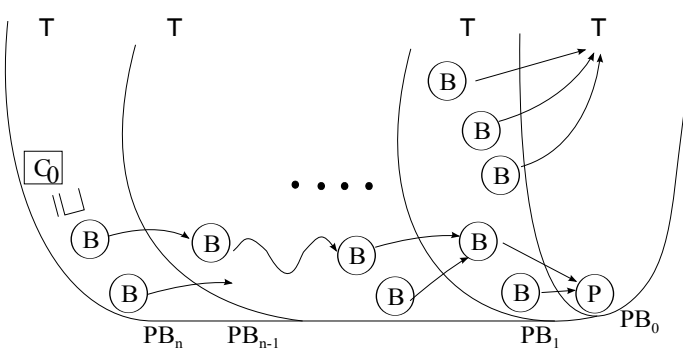

Fig. 4. Computing a Basis for $\operatorname{Pr} e^{*}(\uparrow C)$ or $\operatorname{Pre}^{*}(\uparrow P)$

\section{MODEL CHECKING}

We now use the results of section IV to check business rules on session systems. As mentioned in the introduction, session systems can model business processes, web-based applications, and transactional systems. For these applications, several properties are of huge interest. In this paper, we focus on two classes of interesting properties, namely conflicts of interest, and Chinese Wall Properties.

\section{A. Conflicts of interest}

Conflicts of interest may arise when two clients of a webstore are involved at the same time in an online payment involving the same bank. Similarly, one may not want a bank to deliver its services to competing business entities. We formalize such situations as undesirable patterns, i.e., partial descriptions of undesirable configurations and show that we can check whether such undesired configurations can occur during the lifetime of a system.

Definition 10: A pattern is a graph $(V, E, \tau)$, where $V, E$ and $\tau$ have the same interpretation as in configurations.

We will say that a configuration $C$ matches a pattern $P$ if and only if $P \sqsubseteq C$. Futher, a run $\rho=C_{0} \stackrel{\sigma_{1}}{\longrightarrow} C_{1} \ldots \stackrel{\sigma_{k}}{\longrightarrow} C_{k}$ of a session system meets a pattern $P$ if some configuration $C_{i}$ of the run matches $P$. Finally, a pattern is reachable by a session system $S S$ from a configuration $C_{0}$ iff there exists a run of $S S$ that meets $P$. Thus, checking for a conflict of interest (modeled as a pattern) corresponds to checking if it is possible to reach a configuration which matches the pattern. Note that patterns need not be configurations. However, decision procedures for coverability can be used for patterns, as saturation techniques and proofs for well structure and effectiveness do not use the fact that the considered objects are configurations: $\sqsubseteq$ is defined for any kind of subgraph, and we can set $P \sqsubseteq \top$ for every pattern $P$. Similarly, upward closure of patterns, predecessors, basis, can be defined for patterns, and proved effective. As we restrict ourselves to $k$-bounded configurations, we also restrict to $k$-bounded patterns for a fixed $k$ and denote by $\mathcal{P}^{k}$ the set of $k$-bounded patterns. Now, the results on coverability extend to patterns:

Proposition 11: Given a session system $S S$, a pattern $P \in$ $\mathcal{P}^{k}$, and a configuration $C_{0} \in \mathcal{C}^{k}$, one can decide if there exists a run of $S S$ over $\mathcal{C}^{k}$ starting from $C_{0}$ that meets $P$.

Proof: Following the proof of Corollary 9, one can build a finite sequence $P B^{0}, \ldots, P B^{n}$ of unions of basis, such that $\uparrow P B^{n}=\uparrow p r e^{*}(\uparrow P)$. Using the steps of the construction of $P B^{n}$, we build a directed acyclic graph $G_{P B}$, whose vertices are the bases computed at each step and whose edge relation is given as follows: there is an edge between an element $x \in$ $\left(P B^{j} \backslash P B^{j-1}\right)$ of a basis computed at step $j$ and an element $y \in P B^{j-1}$ computed at step $j-1$ if $\uparrow x \cap \operatorname{Pred}(\uparrow y \backslash \top) \neq \emptyset$, i.e., if $x$ is smaller than an element in $\operatorname{Pred}(\uparrow y \backslash \top)$. We can then find paths from some $B_{0} \in P B^{n}$ to $P$ in $G_{P B}$. We will now show that considering these paths suffices to characterize runs over $\mathcal{C}^{k}$ that meet $P$.

We start by showing that if there is a path in $G_{P B}$ from some $B_{0} \in P B^{n}$ to $P$ then there is a run over $\mathcal{C}$ from $B_{0}$ to a configuration that matches $P$. For every pair $x, y$ in $G_{P B}$, and for every configuration $C_{x} \in \uparrow x$, we know that there exists a configuration $C_{y}$ and a transition $C_{x} \longrightarrow C_{y}$ where $x \sqsubseteq C_{x}, y \sqsubseteq C_{y}$ and $C_{y}$ is a successor of $C_{x}$. Furthermore, by construction, we have $x, y \neq \top$. Hence, if there exists a path $B_{0}, B_{1}, \ldots, B_{k-1}, P$ in $G_{P B}$, then there exists a run $\rho_{B_{0}}$ starting from $B_{0}$ that meets $P$. This immediately implies that if $B_{0} \sqsubseteq C_{0}$, there is a run $\rho_{C_{0}}=C_{0} \longrightarrow C_{1} \longrightarrow C_{2} \longrightarrow$ $\ldots \longrightarrow C_{k}$ starting from $C_{0}$ that meets $P$, such that $k \leq$ $n$, and every $C_{i}$ is greater than an element $B_{i}$ of the graph computed as a temporary basis at step $k-i$.

However, this does not yet imply that the run $\rho_{C_{0}}$ is over $\mathcal{C}^{k}$, as the additional edges in $C_{0} \backslash B_{0}$ may force the run to reach $\top$. But now, since $B_{0} \sqsubseteq C_{0}$, there exists an injective mapping from $B_{0}$ to $C_{0}$, and the number of such injective 
mappings is finite. For a given mapping $\gamma$, we can then compute all consecutive minimal $C_{i}$ 's in the run that modifies only elements that have appeared in the bases computed by the set saturation algorithm. If none of the $C_{i}$ 's occuring along the run meeting $P$ is $\top$, we are done, as we have a witness run from $C_{0}$ to a configuration embedding $P$. On the other hand, if for every $B_{0} \sqsubseteq C_{0}$, every path from $B_{0}$ to $P$ in $G_{P B}$ and every way to embed $B_{0}$ into $C_{0}$, the run towards $P$ encounters a configuration outside $\mathcal{C}^{k}$, then there is no way to meet $P$ in a run over $\mathcal{C}^{k}$. Thus, we can also decide if the run $\rho_{C_{0}}$ in $S S$ corresponding to the path in $G_{P B}$ is in $\mathcal{C}^{k}$.

Conversely, suppose there exists a run $C_{0} \longrightarrow C_{1} \longrightarrow$ $\ldots C_{q}$ with $P \sqsubseteq C_{q}$ over $\mathcal{C}^{k}$. Hence, necessarily, for every $1 \leq i \leq q$, we have $B_{j} \sqsubseteq C_{i}$ for some $B_{j} \in P B^{n}$, as $P B^{n}$ is a basis for all configurations from which a configuration greater than $P$ is accessible. Note that $B_{j} \sqsubseteq C_{i}$ does not imply that $B_{j}$ is computed at step $i$ of the pred-basis computation. However, $B_{j}$ or a greater basis $B_{j^{\prime}}$ must have been computed at step $q-\max \left\{i \mid B_{j} \sqsubseteq C_{i}\right\}$ of the set saturation algorithm, i.e., the graph $G_{P B}$ contains a path that goes through basis (not all of them necessarily appear in the final set $P B^{n}$ ) that are successively computed by the set saturation method. This completes the proof, since we have reduced checking existence of a run meeting $P$ to reachability of $P$ in $G_{P B}$.

Figure 4 illustrates the construction of the pred-basis, and of the graph $G_{P B}$. Circles labeled $B$ represent a temporary basis computed during one step of the algorithm. Edges represent the fact that a temporary basis was computed as a predecessor of a formerly discovered one.

\section{B. Chinese Wall Properties}

Conflicts of interest represent properties of systems at a single instant. Business rules need to guarantee properties through the whole lifetime of a run of a system, that is, may have to consider several situations that can (or should not) appear along a run. For instance, one wants to guarantee that a client receives an artifact sold by a webstore only after a payment. One may also want to ensure non-competition clauses, that is require that an agent involved in an activity should not provide the same service at a later date to a competitor (such properties are also called Chinese Wall Properties, or CWP for short [2]). In both examples, the first idea is to model the properties as a pair of coverability problems: can one reach a pattern $P_{2}$ from a configuration matching $P_{1}$ that is accessible from $C_{0}$ ? This is however a wrong way of modeling these properties: described this way, $P_{1}$ and $P_{2}$ may refer to distinct agents and sessions, while the sales or non-competition examples depict situations involving the same participants. To model such situations, we need to mention which elements in both patterns $P_{1}, P_{2}$ refer to the same agents. This is formalized as follows:

Definition 12: A correlated pattern is a triple $\left(P_{1}, P_{2}, \psi\right)$ where, for $i=1,2, P_{i}=\left(V_{i}, E_{i}, \tau_{i}\right)$ is a pattern and $\psi: V_{1} \rightarrow$ $V_{2}$ is a partial function.

A run $\rho=C_{0} \rightarrow C_{1} \ldots C_{n}$ matches a correlated pattern $\left(P_{1}, P_{2}, \psi\right)$ if there exist $C_{i}$ and $C_{j}$ with $0 \leq i<j \leq n$ and embeddings $h_{1}, h_{2}$ such that $P_{1} \sqsubseteq C_{i}$ via embedding $h_{1}, P_{2} \sqsubseteq C_{j}$ via embedding $h_{2}$ and for all $v \in \operatorname{dom}(\psi)$, $h_{1}(v)=h_{2}(\psi(v))$. In other words, vertices in the two patterns that are connected by $\psi$ must map to the same vertex in corresponding configurations where the two patterns are embedded. A Chinese Wall Property $(C W P)$ is specified by a finite set of correlated patterns $\left\{\left(P_{i}, P_{i}^{\prime}, \psi_{i}\right)\right\}_{i \in 1 \ldots n}$. A session system violates a CWP $\left\{\left(P_{i}, P_{i}^{\prime}, \psi_{i}\right)\right\}_{i \in 1 \ldots n}$. if one of its runs matches one of the correlated patterns $\left(P_{j}, P_{j}^{\prime}, \psi_{j}\right)$. CWPs allow to consider sets of incompatible situations that should not occur: an employee holding a counselor position at time $t$ in a bank $A$ does not have the right to hold the same position at any time greater than $t$, nor to be head of client accounts department for a competitor bank $B$.

In the rest of this section, we will prove that violation of CWPs by a run of a session system over $\mathcal{C}^{k}$ is a decidable problem. More formally:

Theorem 13: Let $S S$ be a session system, $k \in \mathbb{N}$ be a bound on path length in configurations of $S S$. Let $C=$ $\left\{\left(P_{i}, P_{i}^{\prime}, \psi_{i}\right)\right\}_{i \in 1 \ldots n}$ be a CWP. Then, one can decide if there exists a run of $S S$ over $\mathcal{C}^{k}$ violating $C$.

We prove this theorem as follows. First, as we need to identify agents in patterns, we will slightly adapt the semantics of session systems. When an agent is created, its vertex will be attached a tag, that will never be modified, and that will help tracing it along a run. This results in a slight change in the semantics, that preserves WQO and compatibility. Then we will prove that CWP verification amounts to checking two coverability problems with tagged patterns.

Let $T_{\perp}=T \cup \perp$, where $T$ is a finite set of tags. Define an ordering $<$ on $T_{\perp}$ as follows: $\perp<t$ for all $t \in T$. In other words, elements of $T$ are not ordered with respect to each other. A $T$-tagged configuration is a graph $C=(V, E, \tau, T g)$ where $T g \subseteq 2^{T}$ is the set of tags used in $C$, for $v \in V_{A}, \tau(v) \in$ $L \times T_{\perp}$, where $L$ is the set of agent and session labels identified in the untagged case, and labels of vertices in $V_{S}$ and edges in $E$ are defined as in untagged configurations. We extend the ordering $<$ to $L \times T_{\perp}$ such that $(\ell, \perp)<(\ell, t)$ for all $\ell \in L$, and $\left(\ell^{\prime}, t\right)$ and $(\ell, t)$ are incomparable. For a pair of tagged configurations $C_{1}, C_{2}$, we will write $C_{1} \sqsubseteq{ }_{t} C_{2}$ iff there exists a homomorphism $h$ such that for all $v \in V_{1}, \tau_{1}(v)<h\left(\tau_{2}(v)\right)$, and $T g_{1}=T g_{2}$. That is, elements of a configuration with distinct tags from $T$ are incomparable, and configurations with uncomparable sets of used tags are uncomparable too.

Let $T \mathcal{C}^{k}$ be the set of all $T$-tagged configurations whose untagging is in $\mathcal{C}^{k}$ and let $T \mathcal{C}_{\top}^{k}=T \mathcal{C}^{k} \cup \top$. We slightly update the semantics of moves $\rightarrow_{t}$ to take tags into account. The only change is the environment move create. While creating a new agent, the create action now randomly fixes a permanent tag $t$ chosen randomly from $T_{\perp}$ for the new agent, if this tag is not already used, and adds it to the set of tags used. The created agent will carry this tag for the rest of the run: for every $C_{1} \rightarrow C_{2}, v \in V_{1} \cap V_{2}$ and $\tau_{1}(v)=(\ell, t)$ implies that $\tau_{2}(v)=\left(\ell^{\prime}, t\right)$ for some $\ell^{\prime}$. Note that tags play no role in enabling/disabling actions. For a tagged configuration $C_{t}=$ $(V, E, \tau)$, we define its untagged version $U T\left(C_{t}\right)=\left(V, E, \tau^{\prime}\right)$ where for every vertex $v$ in $V_{A}, \tau^{\prime}(v)=\ell$ iff $\tau(v)=(\ell, x)$, and every vertex $v$ in $V_{S}, \tau^{\prime}(v)=\tau(v)$. Untagging easily extends to runs, i.e., for any tagged run $\rho=C_{0} \rightarrow_{t} C_{1} \ldots C_{k}$, $U T(\rho)=U T\left(C_{0}\right) \rightarrow U T\left(C_{1}\right) \ldots U T\left(C_{k}\right)$. We do not change the semantics of patterns, and say that a configuration $C$ embeds a pattern $P$ iff $P \sqsubseteq U T(C)$. We easily get the following result: 
Lemma 14: $\left(T \mathcal{C}_{T}^{k}, \rightarrow_{t}, \sqsubseteq\right)$ is a WSTS. Also, if $C \in T \mathcal{C}_{T}^{k}$, then $\uparrow \operatorname{Pre}(\uparrow C)$ has an effectively computable finite basis.

We can now explain the connection between tags and correlated patterns. Tags will help identify common agents in correlated patterns. We define tagged patterns, i.e., pairs of patterns that carry tags that identify commonalities. Let $\left(P, P^{\prime}, \psi\right)$ be a correlated pattern, with $P=(V, E, \tau), P^{\prime}=\left(V^{\prime}, E^{\prime}, \tau^{\prime}\right)$, and let $T_{\psi}=\left\{t_{v} \mid v \in \operatorname{dom}(\psi)\right\}$. The corresponding $T_{\psi^{-}}$ tagged correlated pattern is $\left(P_{t}, P_{t}^{\prime}, \psi\right)$ where:

- $P_{t}=\left(V, E, \tau_{t}\right)$, where $\tau_{t}(v)=\left(\tau(v), t_{v}\right)$ if $v \in$ $\operatorname{dom}(\psi)$ and $\tau_{t}(v)=(\tau(v), \perp)$ otherwise.

- $\quad P_{t}^{\prime}=\left(V^{\prime}, E^{\prime}, \tau_{t}^{\prime}\right)$ where $\tau_{t}^{\prime}(v)=\left(\tau^{\prime}(v), t_{v}\right)$ if $v \in$ range $(\psi)$ and $\tau_{t}^{\prime}(v)=\left(\tau^{\prime}(v), \perp\right)$ otherwise.

- $\quad \psi(v)=v^{\prime}, \tau_{t}(v)=(\ell, x)$ and $\tau_{t}^{\prime}\left(v^{\prime}\right)=\left(\ell^{\prime}, y\right)$ imply $x=y$.

Using tagged patterns, we can ensure that an agent $v$ identified by a tag $t$ in pattern $P$ of a configuration during an execution is the same agent in the following configuration meeting another pattern $P^{\prime}$. We are now ready to prove decidability of correlated patterns checking:

Lemma 15: There exists a run matching a correlated pattern $\left(P, P^{\prime}, \psi\right)$ from $C_{0}$ iff

(i) there exists $C_{t} \in \operatorname{Pre}^{*}\left(\uparrow P_{t}^{\prime}\right)$ with $P_{t} \sqsubseteq_{t} C_{t}$ in the tagged semantics (using tags from $T_{\psi}$ ).

(ii) $C_{0} \in \operatorname{Pre}^{*}\left(U T\left(\uparrow P_{t} \cap \operatorname{Pre}^{*}\left(\uparrow P_{t}^{\prime}\right)\right)\right)$ with respect to untagged semantics.

This lemma shows equivalence of correlated pattern matching and of pair of coverability problems, and suffices to proove Theorem 13. By Lemma 14, coverability of tagged patterns is effective, so part $i$ ) of Lemma 15 is effective. Similarly, $\uparrow P_{t} \cap \operatorname{Pre} *\left(\uparrow P_{t}^{\prime}\right)$ can be represented by a finite basis, so part ii) of Lemma 15 is also an effective coverability problem.

\section{RElATED WORK}

Several mechanisms and languages have already been proposed to implement or orchestrate sessions into larger applications. A BPEL [5] specification describes a set of independent communicating agents with a rich control structure. Coordination is achieved through message-passing. Interactions are grouped into sessions implicitly through correlations, which specify data values that uniquely identify a session-for instance, a purchase order number. ORC [6] is a programming language for the orchestration of services. It allows algorithmic manipulation of data, with an orchestration overlay to start new services and synchronize their results. ORC has better mechanisms to define workflows than BPEL, but lacks the notion of correlation that is essential to establish sessions among the participants in a service. AXML [7] defines web services as a set of rules for transforming semi-structured documents described, for instance, in XML. However, it does not make workflows explicit, and does not have a native notion of session either. A common feature of these formalisms is that they aim to describe implementations of web services or orchestrations. All of them are Turing powerful, hence properties such as termination of a service, or coverability are undecidable.

The techniques used for session systems use well quasi ordering on graphs, and set a restriction to get wellstructuredness. Within this setting, decidability of coverability is not surprising. Several papers have used graphs transformation systems (GTS) as model and considered verification techniques. [8] studies GTS equipped with the graph minor relation, and show that several subclasses of this model are WSTS. However, the minor relation can not be used in our context to model business rules: a pattern defines actual connections among agents and sessions, and the graph minor, by allowing collapsing of nodes and edges, emphasizes connection among vertices (possibly via paths) rather than edges. Sangnier et al [9] consider reachability and coverability for GTS ordered by subgraph inclusion. Their decidable classes are GTS without deletion rules, context free graph grammars, or grammars with mandatory hyperedge contraction rules. Our model does not fall into these subclasses: sessions can end or be killed, hence deletion can occur, and the join operation merges two edges, hence our model is not context free.

A lot of efforts have also been devoted to services modeling in the $\pi$-calculus community. Session types [10] have been proposed as a formal model for web services, and have then been enhanced to capture various features such as multiple instantiations of identical agents [11] and nested sessions [12]. The main focus is to determine whether an otherwise unconstrained set of processes adheres to the communication discipline specified by a session type. Verification on session address features such as information flow between agents. The expressive power of the whole $\pi$-calculus and session types do not allow for verification of reachability or coverability properties. [13] uses WSTS to show that a fragment of spatial logic is decidable for the fragment of well-typed $\pi$-calculus processes. The considered fragment can express safety properties. A solution to covering problems for $\pi$-calculus with bounded depth have been proposed in [14]. This work shows that for bounded depth $\pi$-calculus, a forward coverability algorithm (EEC) terminates, even if the bound is unknown. There are several similarities between configurations that can be reached by bounded depth $\pi$-calculus terms and $k$-bounded configurations (both can be seen as graphs of bounded path length). Note however that boundedness for configurations of session systems is set as an arbitrary restriction to the semantics. So far, we do not know syntactic restrictions to session systems ensuring a bound on paths length in configurations.

Let us now compare sessions systems and $\pi$-calculus in terms of modeling power. One can see a sessions as a local name shared by its participants. However, there are some features of session systems that do not find a straightforward translation into $\pi$-calculus. First, agents can kill sessions. This feature is essential in many web-based systems, where a server may shut down its activities, and cancel a series of ongoing transactions. In $\pi$-calculus, local names can survive the end of a session, and process are not meant to be aborted. Second, joining a session and querying the system to find an occurrence of an existing session are a non-deterministic actions provided by the environment. Though we think that one could model an environment and simulate killing, querying, and joining with $\pi$-calculus, the semantics of both models appear quite different.

Several variants of $\pi$-calculus have been designed to model services. A variant of ORC and $\pi$-calculus is proposed by [15]. Processes communicate via streams, and choices of a process are implemented as external choices (if-then-else constructs can be implemented this way). This model has an interesting 
expressive power, as it allows to select values from (ordered) streams, minimal elements, first arrived values, etc, which clearly can not be implemented within our session systems. The counterpart of this expressiveness is of course decidability. Implicitly, processes can interact but run until completion, unlike sessions, that can be interrupted. A multiparty session formalism called $\mu-s e$ is proposed by [16]. Its principle is the same as in session systems, that is avoid managing sessions identities explicitly. Sessions in $\mu-s e$ allow participating processes to communicate in a private way, and additional communications are allowed among processes that are located on the same site. Arbitrary numbers of sessions can be created on a site. Communications are handled as usual in $\pi$-calculus. A merging mechanism allows a process to enter a session at any point, and persistent services can be implemented. Session can handle an arbitrary number of joined processes (while session systems define interactions among sets of agents of predetermined sizes). However, the formalism does not provide means to terminate processes before their completion. The CASPIS formalism [17] was influenced by the $\pi$-calculus and by ORC, and designed to orchestrate services. It provides pairwise sessions, modeled as service calls which creates private names shared by the caller and callee of a session, and pipelining, i.e. a way for a service $P$ to call another service $Q$ whenever a new value in produced by $P$. Unlike the preceding variants, CASPIS allows guarded sums (i.e. internal choices), and gives ways to terminate sessions. Conversation types [18] is an extension of $\pi$-calculus that replaces channel based communications by context sensitive message based communications. A conversation is a behavioral type describing multi party interactions among processes. [18] provides typing mechanisms to ensure that conversations are implemented by processes in a compatible way, and that processes can never get stuck when interleaving sessions. A conversation is close to the notion of session in our setting but allows for unbounded number of participants. Like other other $\pi$-calculus variants, this formalism does not allow for abortion of conversations. The COWS approach (see for instance [19]) introduces a complete language for the orchestration of services. COWS allows for definition of stateful services, and proposes correlations variables that implement correlations of messages as in BPEL, a wait operation to suspend processes for a chosen time and a kill operation, that terminates terms within a delimited scope. The semantics of kill does not take into consideration the nature of the canceled terms, while in session systems owners of services (and only them) can select the kind of service to be killed.

\section{CONCLUSION}

This paper has presented a session-based formalism to model systems that handle arbitrary numbers of sessions and agents. A restriction of the semantics of the model to runs over bounded path length configurations allows for decision of coverability problems. An immediate consequence is that checking simple properties such as conflict of interest or Chinese Wall Properties for this restriction is also decidable.

Several issues remain. The first one is efficiency. Our results rely on well-structured systems and set saturation techniques. So far, we do not know the exact complexity of coverability checking for session systems, but complexity of WSTS can be very high, and may need to consider runs of non- elementary lengths. Even with a fixed set of agents (as in [1]), session systems have the expressive power of reset Petri nets, for which coverability is Ackermann-hard [20]. A solution to reduce complexity, and may be avoid the $k$-boundedness restriction could be to rely on abstraction techniques such as the one proposed by [14] to analyze depth-bounded processes. Finally, our model considers finite data, and a possible improvement is to introduce well-structured data in the model, either for variables, or as elements conveyed within sessions.

\section{REFERENCES}

[1] P. Darondeau, L. Hélouët, and M. Mukund, "Assembling sessions," in ATVA, ser. LNCS, vol. 6996, 2011, pp. 259-274.

[2] David F.C. Brewer and Michael J. Nash, "The chinese wall security policy," in EEE SYMPOSIUM ON RESEARCH IN SECURITY AND PRIVACY. IEEE, 1989, pp. 206-214.

[3] A. Finkel and P. Schnoebelen, "Well-structured transition systems everywhere!" Theor. Comput. Sci., vol. 256, no. 1-2, pp. 63-92, 2001.

[4] G. Ding, "Subgraphs and well-quasi-ordering," in Journal of Graph Theory, vol. 16(5), 1992, pp. $489-502$.

[5] T. Andrews, F. Curbera, H. Dholakia, Y. Goland, J. Klein, F. Leymann, K. Liu, D. Roller, D. Smith, S. Thatte, I. Trickovic, and S. Weerawarana, "Business process execution language for web services (BPEL4WS). version 1.1,” 2003. [Online]. Available: http://xml.coverpages.org/BPELv11-May052003Final.pdf

[6] J. Misra and W. Cook, "Computation orchestration," Software and Systems Modeling, vol. 6, no. 1, pp. 83-110, 2007.

[7] S. Abiteboul, O. Benjelloun, I. Manolescu, T. Milo, and R. Weber, "Active XML: A data-centric perspective on web services," in $B D A 02$, 2002.

[8] S. Joshi and B. König, "Applying the graph minor theorem to the verification of graph transformation systems," in Proceedings of CAV 2008, ser. LNCS, vol. 5123, 2008, pp. 214-226.

[9] N. Bertrand, G. Delzanno, B. König, A. Sangnier, and J. Stückrath, "On the decidability status of reachability and coverability in graph transformation systems," in Rewriting Techniques and Applications (RTA'12), ser. LIPIcs, vol. 15, 2012, pp. 101-116.

[10] K. Honda, N. Yoshida, and M. Carbone, "Multiparty asynchronous session types," in POPL, 2008, pp. 273-284.

[11] P.-M. Deniélou and N. Yoshida, "Dynamic multirole session types," in POPL, 2011, pp. 435-446.

[12] R. Demangeon and K. Honda, "Nested protocols in session types," in CONCUR, 2012, pp. 272-286.

[13] L. Acciai and M. Boreale, "Deciding safety properties in infinite-state pi-calculus via behavioural types," in ICALP (2), ser. LNCS, vol. 5556, 2009, pp. 31-42.

[14] T. Wies, D. Zufferey, and T. A. Henzinger, "Forward analysis of depthbounded processes," in FOSSACS, ser. LNCS, vol. 6014, 2010, pp. 94 108.

[15] I. Lanese, F. Martins, V. T. Vasconcelos, and A. Ravara, "Disciplining orchestration and conversation in service-oriented computing," in SEFM. IEEE Computer Society, 2007, pp. 305-314.

[16] R. Bruni, I. Lanese, H. C. Melgratti, and E. Tuosto, "Multiparty sessions in soc," in COORDINATION, ser. LNCS, vol. 5052, 2008, pp. 67-82.

[17] M. Boreale, R. Bruni, R. De Nicola, and M. Loreti, "Sessions and pipelines for structured service programming," in FMOODS, ser. LNCS, vol. 5051, 2008, pp. 19-38.

[18] L. Caires and H. T. Vieira, "Conversation types," Theor. Comput. Sci., vol. 411, no. 51-52, pp. 4399-4440, 2010.

[19] R. Pugliese and F. Tiezzi, "A calculus for orchestration of web services," J. Applied Logic, vol. 10, no. 1, pp. 2-31, 2012.

[20] P. Schnoebelen, "Revisiting ackermann-hardness for lossy counter machines and reset petri nets," in MFCS'10 : Mathematical Foundations of Computer Science, ser. LNCS, vol. 6281, 2010, pp. 616-628. 


\section{APPENDIX}

\section{APPEndiX 1 : Operational Semantics of Session SYSTEMS}

\section{A. Agents operations}

Join: there is an agent $v$, that can execute action $\operatorname{Join}(s, r, c)$ and that is not blocked waiting for a session to be served (i.e. a former bJoin has occured and the created session demand was not merged with an existing session). Variables of $v$ are affected according to the transition. A new session vertex is created and connected to the calling agent. Other vertices, edges and labels remain unchanged.

$$
\begin{aligned}
& \exists v \in V_{A}, \tau(v)=\left(A_{i}, q, v a l\right), \\
& \exists\left(q, g, \operatorname{Join}(s, r, c), q^{\prime}\right) \in \Delta_{i}, \\
& \text { val }=g \wedge \text { val }(\text { blocked })==f f \\
& \tau^{\prime}(v)=\left(A_{i}, q^{\prime}, \text { val }\right) \\
& V_{A}^{\prime}=V_{A} \\
& V_{S}^{\prime}=V_{S} \cup\left\{v^{\prime}\right\}(\text { new vertex }), \\
& E^{\prime}=E \uplus\left\{\left(v,(r, f f), v^{\prime}\right)\right\} \\
& \frac{\tau^{\prime}\left(v^{\prime}\right)=(s, c) \wedge \tau^{\prime}(x)=\tau(x) \text { if } x \notin\left\{v, v^{\prime}\right\}}{(V, E, \tau) \stackrel{\text { Join } \stackrel{(s, r, c)}{\longrightarrow},\left(V^{\prime}, E^{\prime}, \tau^{\prime}\right)}{ }}
\end{aligned}
$$

bJoin: bJoin is similar to Join, with the exception that it blocks the calling agent untill the blocking session demand is served by a merge with an existing session.

$$
\begin{aligned}
& \exists v \in V_{A}, \tau(v)=\left(A_{i}, q, v a l\right), \\
& \exists\left(q, g, \operatorname{Joins}(s, r, c), q^{\prime}\right) \in \Delta_{i} \text {, } \\
& \text { val } \models g \wedge \operatorname{val}(\text { blocked })==f f \\
& \tau^{\prime}(v)=\left(A_{i}, q^{\prime}, v a l_{\mid b l o c k e d:=t t}\right) \\
& V_{A}^{\prime}=V_{A} \\
& V_{S}^{\prime}=V_{S} \cup\left\{v^{\prime}\right\} \text { (new vertex), } \\
& E^{\prime}=E \uplus\left\{\left(v,(r, f f), v^{\prime}\right)\right\} \\
& \frac{\tau^{\prime}\left(v^{\prime}\right)=(s, c) \wedge \tau^{\prime}(x)=\tau(x) \text { if } x \notin\left\{v, v^{\prime}\right\}}{(V, E, \tau) \stackrel{b J o i n(s, r, c)}{\longrightarrow}\left(V^{\prime}, E^{\prime}, \tau^{\prime}\right)}
\end{aligned}
$$

Query Queries allow to query the whole system for the existence of a specific kind of session, with additional constraints on roles. To avoid encoding a zero test with queries, this operation is blocking, that is an agent that queries the system has to wait for a positive answer before continuing.

$$
\begin{aligned}
& \exists v \in V_{A}, \tau(v)=\left(A_{i}, q, \text { val }\right), \\
& \exists\left(q, g, \text { Query }(s, c), q^{\prime}\right) \in \Delta_{i}, \\
& \text { val }=g \wedge \text { val }(\text { blocked })==f f \\
& \tau^{\prime}(v)=\left(A_{i}, q^{\prime}, \text { val }_{\mid \text {blocked }:=t t}\right) \\
& V_{A}^{\prime}=V_{A} \\
& \left.V_{S}^{\prime}=V_{S} \cup\left\{v^{\prime}\right\} \text { (new vertex }\right), \\
& E^{\prime}=E \uplus\left\{\left(v, q r y, v^{\prime}\right)\right\} \\
& \frac{\tau^{\prime}\left(v^{\prime}\right)=(s, c) \wedge \tau^{\prime}(x)=\tau(x) \text { if } x \notin\left\{v, v^{\prime}\right\}}{(V, E, \tau) \stackrel{\text { bJoin(s,r,c) }}{\longrightarrow}\left(V^{\prime}, E^{\prime}, \tau^{\prime}\right)}
\end{aligned}
$$

Spawn: The spawn operation simply creates a new session of the required type. The calling agent is the owner of the created session.

$$
\begin{aligned}
& \exists v \in V_{A}, \tau(v)=\left(A_{i}, q, v a l\right), \\
& \exists\left(q, g, \operatorname{Spawn}(s, r, c), q^{\prime}\right) \in \Delta_{i}, s=\left(N, n_{0}, \delta, R, \Gamma,, l\right) \\
& \text { val }=g \wedge \text { val }(\text { blocked })==f f \\
& V_{A}^{\prime}=V_{A} \\
& V_{S}^{\prime}=V_{S} \cup\left\{v^{\prime}\right\}(\text { new vertex }), \\
& E^{\prime}=E \uplus\left\{\left(v,(r, t t), v^{\prime}\right)\right\} \\
& \tau^{\prime}\left(v^{\prime}\right)=\left(s, n_{0}, c,\{r\}\right) \wedge \tau^{\prime}(v)=\left(A, q^{\prime}, v a l\right) \\
& \wedge \tau^{\prime}(x)=\tau(x) \text { if } x \notin\left\{v, v^{\prime}\right\} \\
& \hline(V, E, \tau) \stackrel{\text { Spawn(s,r,c)}}{\longrightarrow}\left(V^{\prime}, E^{\prime}, \tau^{\prime}\right)
\end{aligned}
$$

Kill(s): The kill operation suppresses all session vertices of a required type owned by the calling agent. Denting by $\Sigma$ the set of all possible edge labels, we have the following rule:

$$
\begin{aligned}
& \exists v \in V_{A}, \tau(v)=\left(A_{i}, q, v a l\right) \\
& \exists\left(q, g, \operatorname{Kill}(s), q^{\prime}\right) \in \Delta_{i} \\
& \text { val } \models g \wedge \text { val }(\text { blocked })==f f \\
& V_{A}^{\prime}=V_{A} \\
& V_{S}^{\prime}=V_{S} \backslash\left\{v^{\prime} \mid \exists\left(v,(r, t t), v^{\prime}\right) \in E\right\} \\
& E^{\prime}=E \cap V^{\prime} \times \Sigma \times V^{\prime} \\
& \frac{\tau^{\prime}(v)=\left(A, q^{\prime}, v a l\right) \wedge \tau^{\prime}(x)=\tau(x) \text { if } x \neq v}{(V, E, \tau) \stackrel{\text { Kill })(s)}{\longrightarrow}\left(V^{\prime}, E^{\prime}, \tau^{\prime}\right)}
\end{aligned}
$$

Agent local update: Local updates change values of variables for a single agent, but may also update agenda. Writing $u p\left(v a l, k_{i}\right)==v^{\prime \prime}$ if the value taken by agenda variable $k_{i}$ is $v^{\prime \prime}$ after applying update $u p$ to valuation $v a l$ we have:

$$
\begin{aligned}
& \exists v \in V_{A}, \tau(v)=\left(A_{i}, q, v a l\right) \\
& \exists\left(q, g, u p, q^{\prime}\right) \in \Delta_{i} \\
& \text { val } \models g \wedge \text { val (blocked })==f f \\
& V_{A}^{\prime}=V_{A} \\
& V_{S}^{\prime}=V_{S} \\
& \left.E^{\prime}=E \backslash \quad\left\{v, k_{i}, v^{\prime}\right\} \mid k_{i} \text { updated }\right\} \\
& \uplus\left\{\left(v, k_{i}, v^{\prime \prime}\right) \mid u p\left(v a l, k_{i}\right)==v^{\prime \prime}\right\} \\
& \frac{\tau^{\prime}(v)=\left(A, q^{\prime}, u p(v a l)\right) \wedge \tau^{\prime}(x)=\tau(x) \text { if } x \neq v}{(V, E, \tau)^{K i l l l(s)}\left(V, E^{\prime}, \tau^{\prime}\right)}
\end{aligned}
$$

\section{B. Transactions operations}

In addition to agents local moves, we have to handle transactions. Transactions are modeled by vertices, and a move in a transaction may result in a relabeling of the transaction's state, but also in changes in the values of variables of agents contributing to the transaction. We disdtinguish two kinds of transaction moves : normal moves, and operations with termination, when the session arrives at its end right after executing an operation. In such case, the corresponding vertex must disappear from the configuration. 
Session operation without termination

$$
\begin{aligned}
& \exists v \in V_{S}, \tau(v)=(S, n, c, J) \\
& s=\left(N, n_{0}, \delta, R, \Gamma, l\right) \\
& \exists\left(n, g, \gamma, a f, n^{\prime}\right) \in \delta \\
& V @ r \models g \tau^{\prime}(v)=\left(S, n^{\prime}, r, c\right) \\
& \forall r \in R, v^{\prime} \in V_{A}\left(v^{\prime},(r, b), v\right) \in E \\
& \wedge \tau\left(v^{\prime}\right)=\left(A_{i}, q, v a l\right), \tau^{\prime}\left(v^{\prime}\right)=\left(A_{i}, q, a f(v a l)\right) \\
& E^{\prime}=E \backslash\left\{\left(v^{\prime}, k_{i}, v^{\prime \prime}\right\} \mid k_{i} \text { updated }\right\} \\
& \backslash\left\{\left(v^{\prime},(r, b), v\right) \mid l(\sigma, r)=\top\right\} \\
& \uplus\left\{\left(v^{\prime}, k_{i}, v^{\prime \prime \prime}\right) \mid a f\left(\bigcup v a l @ R, k_{i}\right)==v^{\prime \prime \prime}\right\} \\
& \qquad(V, E, \tau) \stackrel{\sigma}{\longrightarrow}\left(V, E^{\prime}, \tau^{\prime}\right)
\end{aligned}
$$

Session operation with termination

$$
\begin{aligned}
& \exists v \in V_{S}, \tau(v)=(S, n, c, J) \\
& s=\left(N, n_{0}, \delta, R, \Gamma, l\right) \\
& \exists\left(n, g, \gamma, a f, n^{\prime}\right) \in \delta \wedge \nexists\left(n^{\prime}, g^{\prime}, \gamma^{\prime}, a f^{\prime}, n^{\prime \prime}\right) \in \delta, \\
& V @ r \models g \\
& V_{S}^{\prime}=V_{S} \backslash\{v\} \\
& \forall r \in R, v^{\prime} \in V_{A}\left(v^{\prime},(r, b), v\right) \in E \wedge \\
& \tau\left(v^{\prime}\right)=\left(A_{i}, q, v a l\right), \tau^{\prime}\left(v^{\prime}\right)=\left(A_{i}, q, a f(v a l)\right) \\
& E^{\prime}=E \backslash\left\{\left(v^{\prime}, k_{i}, v^{\prime \prime}\right\} \mid k_{i} \text { updated }\right\} \\
& \backslash\left\{\left(v^{\prime},(r, l), v\right\}\right. \\
& \uplus\left\{\left(v^{\prime}, k_{i}, v^{\prime \prime \prime}\right) \mid a f\left(\bigcup v a l @ R, k_{i}\right)==v^{\prime \prime \prime}\right\} \\
& \frac{(V, E, \tau) \stackrel{\sigma}{\longrightarrow}\left(V^{\prime}, E^{\prime}, \tau^{\prime}\right)}{}
\end{aligned}
$$

\section{C. environment moves}

Agent arrival : Agent arrival in the system is modeled as a simple vertex appedning. As a new agent does not yet take part into anay session, this new vertex is not connected to any session. We denote by $v a l_{\emptyset}$ an initial valuation if which variable blocked is set to $f f$.

$$
\begin{aligned}
& V_{A}^{\prime}=V_{A} \uplus v(\text { new vertex }) \\
& \tau^{\prime}(v)=\left(A, q_{0}, v a l_{\emptyset}\right) \text { for some } A \\
& (V, E, \tau) \longrightarrow\left(V^{\prime}, E, \tau^{\prime}\right)
\end{aligned}
$$

Merge with a blocking demand: When merging a session and a session demand, if the agent asking to join a session is blocked, then joining a session should allow him to procees to next transitions. Unlocking an agent is modeled by changing value of the blocked variable to $f f$. Then, the effect of merge on a configuration graph is to collapse vertices representing sessin and session join demand. To be possible, the constraints attached to the existing session and to the session demand on roles affectation must be satisfiable.

$$
\begin{aligned}
& \exists\left(v_{i},(t, f f), v_{i}^{\prime}\right) \in E, \quad \tau\left(v_{i}\right)=\left(A_{j}, q_{j}, v a l_{j}\right), \\
& \tau\left(v_{i}^{\prime}\right)=(s, n, c, J) \\
& \exists\left(v_{j},(r, f f), v_{j}^{\prime}\right) \in E, \quad \tau\left(v_{j}\right)=\left(A_{j}, q_{j}, v a l_{j}\right), \\
& \tau\left(v_{j}^{\prime}\right)=(s, c) \text {, } \\
& \operatorname{val}_{j}(\text { blocked })==t t
\end{aligned}
$$

Merge with a non-blocking demand

$$
\begin{array}{ll}
\exists\left(v_{i},(t, f f), v_{i}^{\prime}\right) \in E, \quad & \tau\left(v_{i}\right)=\left(A_{j}, q_{j}, v_{a l}\right), \\
& \tau\left(v_{i}^{\prime}\right)=(s, n, c, J) \\
\exists\left(v_{j},(r, f f), v_{j}^{\prime}\right) \in E, \quad & \tau\left(v_{j}\right)=\left(A_{j}, q_{j}, v_{a l}\right), \\
& \tau\left(v_{j}^{\prime}\right)=(s, c), \\
& \operatorname{val}_{j}(\text { blocked })==f f
\end{array}
$$

$c \wedge c^{\prime}$ satisfiable

$\tau^{\prime}\left(v_{i}^{\prime}\right)=\left(S, n, c \wedge c^{\prime}, J \cup\{r\}\right)$,

$\tau^{\prime}(y)=\tau(y)$ for all $y \neq v_{i}^{\prime}$

$E^{\prime}=E \backslash\left\{\left(v_{j},(r, f f), v_{j}^{\prime}\right)\right\} \cup\left\{\left(v_{j},(r, f f), v_{i}^{\prime}\right)\right\}$

$V_{S}=V_{S} \backslash\left\{v_{j}^{\prime}\right\}$

$$
(V, E, \tau) \longrightarrow\left(V^{\prime}, E^{\prime}, \tau^{\prime}\right)
$$

Query Unlocking: When an agent is blocked until a query on an existing session is satisfied, it can be unblocked as soon as a session satisfying the expected type and conditions exists in the system.

$$
\begin{aligned}
& \exists(x,(r, f f), v) \in E \tau(v)=Q \text { uery }(s, c), \\
& \tau(x)=\left(A_{i}, q, \text { val }\right) \wedge \text { val }(\text { blocked })==t t \\
& \exists v^{\prime} \in V_{S}, \tau\left(v^{\prime}\right)=\left(s, n, c^{\prime}, J\right) \\
& c^{\prime} \Rightarrow c \\
& \tau^{\prime}(x)=\left(A_{i}, q, \text { val }_{\text {blocked }}=f f\right), \\
& \tau^{\prime}(y)=\tau(y) \text { for all } y \neq x \\
& E^{\prime}=E \backslash\left\{\left(x,(r, f f), v^{\prime}\right)\right\} \\
& \frac{V_{A}=V_{A} \backslash\{v\}}{(V, E, \tau) \longrightarrow\left(V^{\prime}, E^{\prime}, \tau^{\prime}\right)}
\end{aligned}
$$

\section{APPENDIX 2 : PROOFS}

\section{Well quasi ordering on $\mathcal{C}$}

Proposition 16: $(\mathcal{C}, \sqsubseteq)$ is not a WQO

Proof: $(\mathcal{C}, \sqsubseteq)$ is not a well quasi ordering. Indeed, for a session system with two agent types $\left\{A_{1}, A_{2}\right\}$ and a single session protocol $S=\left(\left\{n_{0}\right\}, n_{0}, \delta=\emptyset,\left\{r_{1}, r_{2}\right\}, \Gamma=\emptyset, l=\emptyset\right)$, one can design an infinite set of configurations $C h=\bigcup_{2<i \in \mathbb{N}} c h_{i}$ such that each $c h_{i}$ is a chain of $i$ vertices and $i-1$ edges such that vertices 1 and $i$ are labeled by $\left(A_{1}, q_{0}\right)$, for every $1<j<i, \tau\left(v_{j}\right)=\left(A_{2}, q_{0}\right)$, and for every pair of vertices $v_{i}, v_{i+1}$, there exists a session vertex $v_{s, i, i+1}$ labeled $\left(s, n_{0}, \emptyset\right)$ and a pair of edges $e_{i, s}=\left(v_{i},(1, t t), v_{s, i, i+1}\right)$ and $e_{s, i+1}=$ $\left(v_{s, i, i+1},(2, f f), v_{i+1}\right.$ such that $\tau\left(h_{i}\right)=\left(s, n_{0}, \emptyset\right)$. Clearly, for any pair of distinct chains $c h_{i}, c h_{j}$ in $C h$, we have $c h_{i} \nsubseteq c h_{j}$ and $c h_{j} \nsubseteq c h_{i}$. This means that $(\mathcal{C}, \sqsubseteq)$ contains an infinite antichain, and is hence not a well-quasi order. Examples of such chains are provided in figure 5

\section{E. Proof of Theorem 7}

To prove well structuredness of $\left(\mathcal{C}_{\top}^{k}, \longrightarrow\right.$, $)$, we first need the following lemma:

Lemma 17: For a fixed $k \in \mathbb{N},\left(\mathcal{C}_{\top}^{k}, \sqsubseteq\right)$ is a WQO.

Proof: Let us first show that $\left(\mathcal{C}^{k}, \sqsubseteq\right)$ is a WQO. For this, it suffices to show that $\sqsubseteq$ is well-founded and does not contain infinite antichains. We can easily see that for a given configuration $C$, there exists only a finite number of configurations (up to isomorphisms) that are subgraphs of $C$. All these configurations that are not isomorphic to $C$ have 


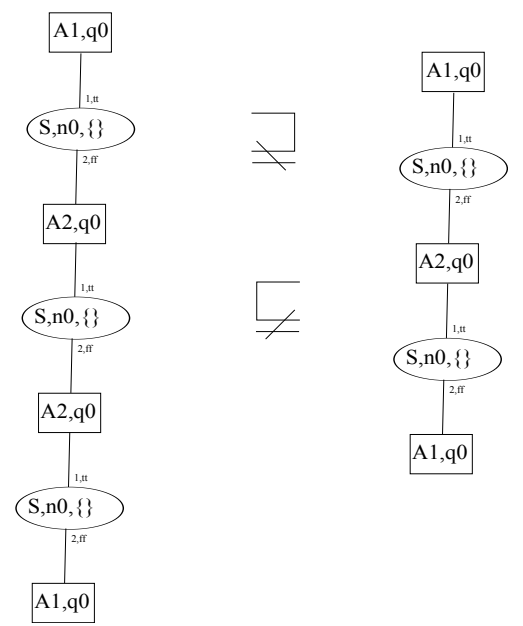

Fig. 5. Configurations are not Well quasi ordered by $\sqsubseteq$

less vertices, or less edges. Hence, there exists only finite decreasing sequences of configurations, and $\sqsubseteq$ is well-founded.

We can now show that $\mathcal{C}^{k}$ contains only finite antichains, i.e., sets of pairwise incomparable elements. For this, we will reuse results from [4], that shows that for any $k$, labeled graphs of $k$-bounded path length, ordered by the induced subgraph relation is a WQO. For two graphs $G_{1}, G_{2}$ one says that $G_{1}$ is a induced subgraph of $G_{2}$, denoted $G_{1} \leq G_{2}$ iff $G_{1}$ has exactly the edges that appear in $G_{2}$ over isomorphic vertex set. A graphs is in $\mathcal{P}_{n}$ (of path length bounded by $n$ ) if it does not contain simple sequences of edges of length greater than $n$. The result established by [4] is that $\left(\mathcal{P}_{k}, \leq\right)$ is a WQO. The proof is shown for undirected graphs, but easily extends to directed graphs, as one can simulate edge orientation with additional edges and vertices. This transforms a directed graph $G=(V, E)$ into a undirected graph $G^{\prime}=\left(V \cup V_{E}, E_{1} \cup E_{2}\right)$, in which, for every edge $e=\left(v, l, v^{\prime}\right) \in E, V_{E}$ contains a vertex $v_{e}, E_{1}$ contains an edge $\left(v\right.$, org,$\left.v_{e}\right)$ and $E_{2}$ contains an edge $\left(v_{e}, l, v^{\prime}\right)$. Let us denote by $\eta$ this mapping from directed graphs to undirected ones.

Configuration of $\mathcal{C}^{k}$ are directed graphs, that can be transformed into undirected graphs whose path length is bounded by $2 . k$. For a pair of configurations $C_{1}, C_{2}$ and a pair of graphs $G_{C_{1}}=\eta\left(C_{1}\right)$ and $G_{C_{2}}=\eta\left(C_{2}\right), G_{C_{1}} \leq G_{C_{2}}$ implies $C_{1} \sqsubseteq C_{2}$ (though the converse is not true, in general, as graph inclusion allows more edges in $C_{2}$ ). This means that $C \nsubseteq C^{\prime}$ implies $G_{C} \not \leq G_{C^{\prime}}$.

Consider any antichain $X$ in $\left(\mathcal{C}^{k}, \sqsubseteq\right)$. The image of this set of incomparable elements w.r.t to $\sqsubseteq$ via $\eta$ is a set of graphs in $\mathcal{P}_{2 . k}$ that are incomparable w.r.t. $\leq$. Since $\left(\mathcal{P}_{n}, \leq\right)$ is a WQO for any $n$, its antichains are of bounded size, and hence, the size of antichains in $\left(\mathcal{C}^{k}, \sqsubseteq\right)$ is also bounded. Hence $\left(\mathcal{C}^{k}, \sqsubseteq\right)$ is also a WQO.

Adding $T$ to the set of configurations keeps well-quasi ordering. First, the size of decreasing chains remains finite, as chains starting from $T$ simply extend chains in $\mathcal{C}^{k}$ by one element. Finiteness of antichains is also preserved: consider any antichain $X$ in $\left(\mathcal{C}_{\top}^{k}, \sqsubseteq\right)$. We cannot have $\top \in X$ since $C \sqsubseteq \top$ for every $C \in \mathcal{C}_{\top}^{k}$. Hence $X \subseteq \mathcal{C}^{k}$ and the size of $X$ must be bounded by the previous result.
Theorem 7 : For a fixed $k \in \mathbb{N},\left(\mathcal{C}_{\top}^{k}, \longrightarrow, \sqsubseteq\right)$ is a wellstructured transition system.

Proof: From Lemma 17, we know that $\left(\mathcal{C}_{\top}^{k}, \sqsubseteq\right)$ is a WQO. So it remains to show that the transition relation $\longrightarrow$ is compatible with $\sqsubseteq$. Let $\left(C_{1}, C_{1}^{\prime}\right) \in \longrightarrow$ and let $C_{1} \sqsubseteq C_{2}$. If $C_{1}=\top$, then we also have $C_{1}^{\prime}=C_{2}=\top$, and hence, by definition of $\top$ any action enabled in $C_{1}$ is also enabled in $C_{2}$. If $C_{1} \neq \top$, then the move from $C_{1}$ to $C_{1}^{\prime}$ can be an internal action $a$ of a session $s$, an agent operation $\sigma$, or an environment move (arrival of agent or merge). It is then easy to see that any move at $C_{1}$ that creates a configuration in $\mathcal{C}^{k}$ can also be executed by $C_{2}$ since guards of this move only consider elements of $C_{1}$ and their labels, which have an image in $C_{2}$.

The move from $C_{2}$ to some $C_{2}^{\prime}$ can violate the $k$-boundedness assumption, i.e, we can have $C_{2}^{\prime}=\top$, but as we have $C_{1}^{\prime} \sqsubseteq \top$, compatibility is ensured for such situations. Now if $C_{1}$ performs a move that violates $k$-boundedness, i.e. reaches $\top$, then, as all edges and vertices of $C_{1}$ have an image in $C_{2}, C_{2}$ also moves to $\top$ configuration by creating a path of lenght greater than $k$ among vertices that are the image on $C_{1}$. And then, by definition, as we have $C_{1}^{\prime} \sqsubseteq \top$, compatibility also holds for this situation.

Now, we have to consider pairs of configurations performing the same move from $C_{1}$ to $C_{1}^{\prime}$ and $C_{2}$ to $C_{2}^{\prime}$ where all these configrations are not $T$. This move can be an internal action $a$ of a session $s$, an agent operation $\sigma$, or an environment move (arrival of agent or merge). Now, let $C, C^{\prime}$ be two configurations in $\mathcal{C}^{k}$ such that $C \sqsubseteq C^{\prime}$. Let $t(C)$ be a configuration obtained by relabeling vertices or edges of $C$, deleting/adding edge or vertices, and let us call $t($. this transformation. Then the same operation performed on the image of $C$ (i.e a subgraph of $C^{\prime}$ ) in $C^{\prime}$ via $\psi$ results in a configuration $t\left(C^{\prime}\right)$ such that $t(C) \sqsupseteq t\left(C^{\prime}\right)$. The effects of session actions, agents internal actions can all be encoded as a finite sequence of such transformations that will preserve $\sqsubseteq$.

Let us first consider session actions. If $a$ is not the last action of the session $s$ modeled by a session vertex $v$, then executing $a$ results in a relabeling of vertex $v$, and possibly a relabeling of the set of vertices $V_{v}$ connected to $v$ symbolizing agent contributing to $s$ if some variables are updated, a change in their references, that is a reorganization of connections among vertices of $V_{v}$ and vertices connected to $V_{v}$ via modeling of the references, denoted $\operatorname{Links}\left(V_{v}\right)$. As $C_{1} \sqsubseteq C_{2}$, all vertices of $V_{v} \cup \operatorname{Links}\left(V_{v}\right)$ have an image in $C_{2}$ and similarly all edges connected to $V_{v}$ have an image in $C_{2}$. This image preserves labeling of vertices, i.e variables valuations, hence all guards that hold on a subset of variables in $C_{1}$ also hold for their image in $C_{2}$. Hence, local action $a$ can also be performed from $C_{2}$, which results in a new configuration $C_{2}^{\prime}$. As $C_{1} \sqsubseteq C_{2}$, there exists a morphism $\psi$ from $C_{1}$ to $C_{2}$. Considering the part of the subgraph that is rewritten by execution of action $a$, one can easily check that all vertices in $V_{v} \cup \operatorname{Links}\left(V_{v}\right) \cap C_{1}$ and their image in $C_{2}$ by $\psi$ change their values and connections accordingly, hence $\psi$ is still a morphism from $C_{1}^{\prime}$ to $C_{2}^{\prime}$ and we have $C_{1}^{\prime} \sqsubseteq C_{2}^{\prime}$.

If $a$ is the last action of the session, executing this action results in disappearance of the vertex $v$ modeling session $s$, which has an image in $C_{2}$, and hence this action can also be executed from $C_{2}$, and results in a similar session vertex 
deletion, and the restriction of $\psi$ to remaining edges and vertices ensures $C_{1}^{\prime} \sqsubseteq C_{2}^{\prime}$.

Environments moves that merge session demands and exisiting sessions collapse existing session vertices, which can also be modeled as a finite sequence of configuration transformations: deletion of the demand, connection of the joining agent in the demanded role, and relabeling of the session vertex to take into account constriants of the joining agent. Agents arrival results in the creation of a new vertex, and is not guarded, and hence can be applied similarly to any pair of configurations $C, C^{\prime}$ by adding one new vertecs $v$ to $C$ and $v^{\prime}$ to $C^{\prime}$. It then suffices to create a new morphism $\psi \cup\left[v, v^{\prime}\right)$ to define an embedding from $C \cup\{v\}$ to $C^{\prime} \cup\left\{v^{\prime}\right\}$.

The only remaining action is the $\operatorname{kill}(s)$ operation, which kills all sessions of a specific kind owned by the agent execution this operation. Killing sessions in a configuration resumes again to edges and vertices deletion, but in a pair of configurations $C \sqsubseteq C^{\prime}$, the number of edges to be deleted is not necessarily the same. However, guard of kill actions only depend on the local variables and references of the agent $v$ executing the kill operation, that is on its labeling and connection within $\operatorname{Links}(v)$. Hence, if a guard $g$ for a killing operation holds on $C$, it holds on $C^{\prime}$. The result of the killing produces a new configuration, with the same number of agent vertices, which is a projection $\pi_{s}($.$) of the initial configuration$ on all agent vertices plus session vertices either not owned by $v$ or that are not of the deleted type (plus a relabeling of the agent killing its sessions). Hence, if $C \sqsubseteq C^{\prime}$, the restriction of the embedding relation $\psi$ from $C$ to $\bar{C}^{\prime}$ to projected vertices and edges is still an embedding from $t\left(\pi_{\mathcal{s}}(C)\right.$ to $t\left(\pi_{\mathcal{s}^{\prime}}\left(C^{\prime}\right)\right.$.

Hence, $\sqsubseteq$ is compatible with $\longrightarrow$.

\section{F. Proof of lemma 8}

Before proving that a pred-basis can be effectively computed for any upward-closed set represented by its basis, we first show the following lemma that addresses the same problem for a single configuration.

Lemma 18: Let $C \in \mathcal{C}_{\top}^{k}$. Then one can compute effectively a finite basis for $\uparrow \operatorname{Pre}(\uparrow C)$.

Proof: First of all, for two arbitray sets of configurations $X$ and $X^{\prime}$, we have $\uparrow\left(\operatorname{Pre}\left(X \cup X^{\prime}\right)\right)=\uparrow(\operatorname{Pre}(X)) \cup$ $\uparrow\left(\operatorname{Pre}\left(X^{\prime}\right)\right)$. Hence, we can consider $k$-bounded configurations and $\top$ separately, and we have $\uparrow(\operatorname{Pre}(\uparrow(C)))=$ $\uparrow\left(\operatorname{Pre}\left(\uparrow(C) \cap \mathcal{C}^{k}\right)\right) \cup \uparrow(\operatorname{Pre}(\top))$.

One may also notice that if we restrict our action alphabet to operations that are not killing sessions, there exists only a finite set of configurations that can reach $C$ via a single move in $\longrightarrow$. We will denote by $\operatorname{pre}_{\text {nokill }}(C)$ this set of configurations. Conversely, if one can reach $C$ by a killing operation, then set of configurations preceding $C$ by a kill move is infinite. These configurations differ, depending on the number of killed sessions, and on the agent that has performed the kill operation. This agent is represented by a particular vertex $v$ appearing in $C$, so there is only a finite number of agants that may have performed a kill. Similarly, there exists only a finite number of kinds of sessions (not more than the number of session protocols) that can be killed. So, for a fixed vertex $v$ and a fixed session protocol $s$, the set of configurations preceding $C$ may have an arbitrary number of sessions of type $s$ owned by $v$, and connected to other agents in $C$. However these sets of configurations can be represented by their minimal elements, which are configurations in which no session of type $s$ owned by $v$ existed. That is, denoting by $\operatorname{pre}_{k i l l}(C)$ the set of configurations preceding $C$ by a kill operation, if $C=(V, E, \tau)$ then the finite set

$$
\left\{\begin{aligned}
& \exists v \in V_{A}, \tau(v)=\left(A_{i}, q^{\prime}, v a l\right) \\
C^{\prime}=\left(V, E, \tau^{\prime}\right) \mid & \wedge\left(q, g, \operatorname{Kill}(s), q^{\prime}\right) \in \Delta_{i} \\
& \wedge v a l \models g \wedge \tau^{\prime}(v)=\left(A_{i}, q, v a l\right) \\
& \wedge \tau(x) \text { for } x \neq v
\end{aligned}\right\}
$$

is a basis for $\operatorname{pre}_{k i l l}(C)$. More intuitively, agents that may have performed a kill perform a step backward without changing the number of sessions (i.e the step backward relabels an agent without adding session vertices nor edges). Note that an agent has a predecessor configuration via a $\operatorname{kill}(s)$ operation only when it does not own sessions of type $s$ in $C$.

Let us now consider the set of predecessors of $C$ via operation that are not kills, denoted $\operatorname{pre}_{\text {nokill }}(C)$. The effect of a backward move for each operation of this kind can be modeled as a finite sequence of vertices relabeling (sessions internal action, agents affectations), addition of a session vertex and assocated edges (session final action), addition of a session vertecs, and of an agent (when changing the valuation of an agent is done via an existing session) or a modification of a finite set of session vertices and edges of $C$ (session and session demand merge), removal of a single vertex (agent arrival), removal of a session vertex and associated edge (session spawn or join demand). Clearly, $\operatorname{pre}_{\text {nokill }}(C)$ is finite. When $C \sqsubseteq C^{\prime}$, that is when a morphism $\psi$ from $C$ to $C^{\prime}$ exists, each sequence of graph transformation leading from $C$ to a configuration $C_{\text {pre }} \in$ pre $_{\text {nokill }}(C)$ can be applied to $C^{\prime}$ up to renaming of vertices via $\psi$, and leads to a configuration $C_{\text {pre }}^{\prime} \in \operatorname{pre}_{\text {nokill }}\left(C^{\prime}\right)$ such that $C_{\text {pre }} \sqsubseteq C_{\text {pre }}^{\prime}$. Hence, the partial ordering $\sqsubseteq$ is preserved by $\operatorname{pre} e_{\text {nokill }}()$ in $\mathcal{C}^{k}$, and $\operatorname{pre}_{\text {nokill }}(C)$ is a basis for $\uparrow \operatorname{pre}_{\text {nokill }}(\uparrow C \backslash \top)$

Let us first define $\operatorname{Pre}(\top)$. As explained before, one can reach configuration $\top$ from $T$, or from a configuration $C$ does not yet contain paths of length greater than $k$. Configuration $C$ is a configuration in which merging a session demand and an existing session, or learning the address of another agent can create a path of length greater than $k$. That is such configurations are graphs that either:

- $\quad$ contain two paths $\rho_{1}, \rho_{2}$ respectively of length $x$ and $y$ in separate connected components, such that $x \leq k$, $y \leq k, x+y \geq k$ and the last vertex in path $\rho_{1}$ is attached to a join demand for a session $s$, with constraints $c_{1}$, the first vertex in path $\rho_{2}$ is involved in a session $s$ with constraints $c_{2}$, and $c_{1} \wedge c_{2}$ is satisfiable. Let us denote $G_{1, k}$ the set of configurations that contain exactly two paths of this form and nothing else. Obviously, $G_{1, k}$ is finite.

- $\quad$ one path of length $k$ in a connected component such that one vertex (agent) of the path "knows an agent $A$ as the $i^{t h}$ entry of its references, that is not yet in the path, and is involved in a session with the last vertex of the path that can execute an action that affect this 
entry to an entry of the last agent of the path. Let us denote by $G_{2, k}$ the set of configurations that contain only a path of this from. Here again, there exists only a finite number of such chains of length $k$.

Every graph in $\mathcal{C}_{\top}^{k}$ which contains more elements than a graph in $G_{1, k}$ is either $\top$, from which any move goes to $\top$, or another graph $C$ larger than some configuration in $G_{1, k}$ (more $k$-bounded connected components, more edges and sessions), from which there exists an action that creates a path of length at least $k+1$ (that is there exists a move from $C$ to $\top$ ). Similar remark holds for elements of $G_{2, k}$. Hence $\uparrow\left(G_{1, k} \cup G_{2, k}\right)=$ $\operatorname{Pre}(\top)$.

We can now show, simply by rewriting $\uparrow(\operatorname{Pre}(\uparrow(C)))$ that its basis is effectively computable. We denote by $\min (X)$ the set of minimal elements of $X$ w.r.t. $\sqsubseteq$ :

$$
\begin{aligned}
& \operatorname{Basis}(\uparrow(\operatorname{Pre}(\uparrow(C)))=\min (\uparrow(\operatorname{Pre}(\uparrow(C))) \\
& =\min (\uparrow(\operatorname{Pre}(\uparrow(C) \backslash \top) \cup \uparrow(\operatorname{Pre}(\top)) \\
& =\min \left(\uparrow\left(\operatorname{Pre}(\uparrow(C) \backslash \top) \cup \uparrow\left(G_{1, k} \cup G_{2, k}\right)\right)\right. \\
& =\min \left(\begin{array}{l}
\uparrow\left(P r e_{k i l l}(\uparrow(C) \backslash \top)\right) \cup \uparrow\left(\operatorname{Pre}_{\text {nokill }}(\uparrow(C) \backslash \top)\right) \\
\cup \uparrow\left(G_{1, k}\right) \cup \uparrow\left(G_{2, k}\right)
\end{array}\right) \\
& =\min \left(\begin{array}{ll} 
& \min \left(\uparrow\left(\operatorname{Pre}_{k i l l}(\uparrow(C) \backslash \top)\right)\right) \\
\cup & \min \left(\uparrow\left(\operatorname{Pre}_{\text {nokill }}(\uparrow(C) \backslash \top)\right)\right) \\
\cup & \min \left(\uparrow\left(G_{1, k}\right)\right) \cup \min \left(\uparrow\left(G_{2, k}\right)\right)
\end{array}\right) \\
& =\min \left(\operatorname{Pre}_{k i l l}(\uparrow(C)) \cup \min \left(\operatorname{Pre}_{\text {nokill }}(\uparrow(C)) \cup G_{1, k} \cup G_{2, k}\right)\right.
\end{aligned}
$$

As any of the terms in the minimization is an effectively computable set, and as $\sqsubseteq$ is effective, then a basis for $\uparrow(\operatorname{Pre}(\uparrow(C))$ is effectively computable.

We are now ready to prove lemma 8 .

Lemma 8 Let $X$ be an upward closed subset of $\mathcal{C}_{\text {T }}^{k}$ represented by it basis $\left\{B_{1}, B_{2}, \ldots, B_{n}\right\}$. Then we can effectively compute a basis for $\uparrow \operatorname{Pre}(X)$.

Proof: By Lemma 18, for each $B_{i}$, we can compute a basis for $\uparrow \operatorname{Pre}\left(\uparrow\left\{B_{i}\right\}\right)$. Then, a basis for $\uparrow \operatorname{Pre}(X)$ is the set of minimal elements in $\bigcup_{i=1}^{n} \operatorname{Basis}\left(\uparrow \operatorname{Pre}\left(\uparrow\left\{B_{i}\right\}\right)\right)$.

\section{G. Proof of corollary 9}

Corollary 9 For a sessions system $S=(\mathcal{A}, \mathcal{S})$, coverability of a configuration $C$ by a run over $\mathcal{C}_{\top}^{k}$ from an initial configuration $C_{0}$ is decidable.

Proof: We show that there is an effective algorithm to check, for a given configuration $C$ and a session system $S$, if there exists a run of $S$ starting from a configuration $C_{0}$ that reaches a configuration that subsumes $C$.

We start from $C$. We know that $\{C\}$ is a basis for $\uparrow C$. Furthermore, any run that ends in a configuration in $\uparrow C$ is a run that covers $C$. We are hence looking for a run that starts from $C_{0}$ and reaches a configuration in $\uparrow C$. Such a run exists iff $C_{0} \in \uparrow p r e^{*}(\uparrow C)$, or equivalently, if $C_{b} \sqsubseteq C_{0}$ for some $C_{b} \in \operatorname{Basis}\left(\uparrow p r e^{*}(\uparrow C)\right)$. As $\left(\mathcal{C}_{\top}^{k}, \longrightarrow, \sqsubseteq\right)$ is a WSTS (by Theorem 7) with effective $\sqsubseteq$ and effective pred basis computation (by Lemma 8), one can use a set-saturation algorithm to compute a basis for $\uparrow p r e^{*}(\uparrow C)$. The algorithm to compute a basis $P B=\operatorname{Basis}\left(\uparrow p r e^{*}(\uparrow C)\right)$ is a fixpoint algorithm. We start from $P B_{0}=\operatorname{Basis}(\uparrow C)=\{C\}$, and then compute iteratively $P B^{k}=P B^{k-1} \cup \operatorname{Basis}\left(\uparrow p r e\left(\uparrow P B^{k-1}\right)\right)$, and stop as soon as $\uparrow P B^{k}=\uparrow P B^{k-1}$. It has been proved in [3] that for any upward closed set $X$, this algorithm is correct and terminates when the pred-basis computation is effective. Hence, at the end of the fixpoint computation, we have $\uparrow \bigcup P B^{k}=\operatorname{Pre} e^{*}(\uparrow)$.

Now, for any element $C_{b}$ in $P B$, there exists a run $\rho_{b}$ from $C_{b}$ to a configuration $C_{b}^{\prime}$ that covers $C$. Hence, if $C_{b} \sqsubseteq C_{0}$ there exists a run over the same actions than $\rho_{b}$ from $C_{0}$ to a configuration greater than $C_{b}^{\prime}$, and hence greater than $C$. As $\sqsubseteq$ is effective, it then suffices to build the basis $P B$, and compare its elements to $C_{0}$ to check if $C$ is coverable from $C_{0}$.

\section{H. Proof of Lemma 14}

Lemma $14\left(T \mathcal{C}_{\top}^{k}, \rightarrow_{t}, \sqsubseteq\right)$ is a WSTS. Also, if $C \in T \mathcal{C}_{\top}^{k}$, then $\uparrow \operatorname{Pre}(\uparrow C)$ has an effectively computable finite basis.

Proof: $\left(T \mathcal{C}_{+}^{k}, \sqsubseteq\right)$ is a WQO follows from the fact that $\left(\mathcal{C}_{\tau}^{k}, \sqsubseteq\right),\left(T_{\perp},<\right)$ are WQOs and $\left(2^{T}, \subseteq\right)$ is a finite set and that the product of two WQO or a product of a WQO with a finite set of elements are WQOs too (products only increase the number of incomparable elements).

Next, $\left(T \mathcal{C}_{\top}^{k}, \rightarrow_{t}\right)$ simulates $\left(\mathcal{C}_{\top}^{k}, \rightarrow_{t}\right)$, i.e if $C_{1}$ is a tagged configuration, $C_{1}^{\prime}=U T\left(C_{1}\right)$ and $C_{1}^{\prime} \stackrel{\sigma}{\rightarrow} C_{2}^{\prime}$, then there exists a tagged configuration $C_{2}$ such that $C_{1} \stackrel{\sigma_{\rightarrow}}{\rightarrow} C_{2}$ and $U T\left(C_{2}\right)=C_{2}^{\prime}$. Indeed, the only modification in the tagged semantics concerns agents creation. If $C_{1}$ is of the form $C_{1}=\left(V_{1}, E_{1}, \tau_{1}, T g_{1}\right)$, and if $C_{1}^{\prime} \stackrel{\text { create }}{\longrightarrow} C_{2}^{\prime}$, then there exists a move from $C_{1}$ that creates an agent with tag $\perp$, even if all tags have already been used (i.e. when $T g_{1}=T$ ). Now, suppose $C_{1} \sqsubseteq C_{1}^{\prime}$ and $C_{1} \stackrel{\sigma}{\rightarrow} C_{2}$. Then $U T\left(C_{1}\right) \sqsubseteq U T\left(C_{1}^{\prime}\right)$ and $U T\left(C_{1}\right) \stackrel{\sigma}{\rightarrow} U T\left(C_{2}\right)$. Since the untagged system is a WSTS, there is a configuration $C_{2}^{\prime \prime}$ such that $U T\left(C_{1}^{\prime}\right) \rightarrow C_{2}^{\prime \prime}$ and $U T\left(C_{2}\right) \sqsubseteq C_{2}^{\prime \prime}$. Using the simulation property shown above, we can find $C_{2}^{\prime}$ such that $C_{1}^{\prime} \rightarrow C_{2}^{\prime}, U T\left(C_{2}^{\prime}\right)=C_{2}^{\prime \prime}$ and $C_{2} \sqsubseteq C_{2}^{\prime}$. Hence, $\left(T \mathcal{C}_{\top}^{k}, \rightarrow_{t}, \sqsubseteq\right)$ is a WSTS.

To show effectiveness of pred-basis, as for untagged configurations, we can list the set of predecessors of a given configurations, and find a finite basis for sets of configurations that can reach $T$ in one move. The only backward move that differs with respect to proof of proposition 18 is the create move. However, this backward move is deterministic, and leads to a finite basis: The tag to be used during a creation is determined by the tag of the created agent in the reached configuration. If the agent to remove has tag $i$, then the only creation action leading to addition of this agent is a creation with tag $i$. All other cases preserve agent tags, and have the same effect and properties as in the proof of Lemma 18.

\section{Proof of Theorem 13}

Lemma 15: There exists a run matching a correlated pattern $\left(P, P^{\prime}, \psi\right)$ from $C_{0}$ iff

(i) there exists $C_{t} \in \operatorname{Pre}^{*}\left(\uparrow P_{t}^{\prime}\right)$ with $P_{t} \sqsubseteq t C_{t}$ in the tagged semantics (using tags from $T_{\psi}$ ).

(ii) $C_{0} \in \operatorname{Pre}^{*}\left(U T\left(\uparrow P_{t} \cap \operatorname{Pre}^{*}\left(\uparrow P_{t}^{\prime}\right)\right)\right)$ with respect to untagged semantics.

Proof: We proceed by showing the implication in the two directions. $\Rightarrow$ : Let $\rho$ be a run that matches $\left(P, P^{\prime}, \psi\right)$, 
i.e., $\rho=C_{0} \longrightarrow \ldots C_{i} \ldots \longrightarrow C_{j}$ is such that $P \subseteq C_{i}$, $P^{\prime} \subseteq C_{j}$, and such that the agents matched by $P$ and $P^{\prime}$ in $\bar{C}_{i}$ and $C_{j}$ coincide, i.e., there exists an embedding $h$ from $P$ to $C_{i}$, an embedding $h^{\prime}$ from $P^{\prime}$ to $C_{j}$, and for every vertex $v \in \operatorname{dom}(\psi), h^{\prime}(\psi(v))=h(v)$. One can easily transform a run from the untagged semantics into a tagged run where agents carry tags by non-deterministically choosing tags at agents creation time. This means that in the tagged semantics, there exists a run of the form $\rho^{t}=C_{0}^{t} \ldots C_{i}^{t} \ldots C_{j}^{t}$ such that $P_{t} \sqsubseteq C_{i}^{t}$ and $P_{t}^{\prime} \sqsubseteq C_{j}^{t}$, where $P_{t}$ and $P_{t}^{\prime}$ are the tagged patterns derived from $\left(P, P^{\prime}, \psi\right)$. Hence, we have $C_{i}^{t} \in \operatorname{pre}^{*}\left(C_{j}^{t}\right)$, which implies $C_{i}^{t} \in \operatorname{pre} e^{*}\left(\uparrow P_{t}^{\prime}\right)$, and hence $C_{i}^{t}$ is the witness for property $i)$. We also have $C_{0}^{t} \in \operatorname{pre}^{*}\left(C_{i}^{t}\right)$ and $C_{i}^{t} \in \uparrow\left(P_{t}\right) \cap$ pre $e^{*}\left(\uparrow P_{t}^{\prime}\right)$. By removing tags, we get $C_{0} \in \operatorname{pre}^{*}\left(U T\left(\uparrow\left(P_{t}\right) \cap\right.\right.$ pre $\left.\left.e^{*}\left(\uparrow P_{t}^{\prime}\right)\right)\right)$, i.e, condition $\left.i i\right)$ is satisfied.

$\Leftarrow$ : Suppose $i$ ) and $i i)$ are satisfied. Then, there exists $C_{t}$ such that $P_{t} \sqsubseteq_{t} C_{t}$, and $C_{t} \in \operatorname{pre} e^{*}\left(\uparrow P_{t}^{\prime}\right)$. Hence, we know that $\uparrow\left(P_{t}\right) \cap \operatorname{Pre}^{*}\left(\uparrow P_{t}^{\prime}\right)$ is not empty. Now suppose that $C_{0} \in \operatorname{Pre}^{*}\left(U T\left(\uparrow\left(P_{t}\right) \cap p r e^{*}\left(\uparrow P_{t}^{\prime}\right)\right)\right)$. It means that there exists a run from $C_{0}$ to a configuration $C_{i}$, such that $C_{i} \in U T\left(\uparrow\left(P_{t}\right) \cap \operatorname{Pre}^{*}\left(\uparrow P_{t}^{\prime}\right)\right)$, as the tagged semantics allows to associate arbitrary tags to agents at creation time. Hence, if $C_{i}$ can be reached from $C_{0}$, there exists a way to tag $C_{0}$ arbitrarily and to attach tags to agents in $C_{i}$ at creation time in such a way that $C_{i}=U T\left(C_{i}^{t}\right)$ for some tagged configuration $C_{i}^{t}$ in $\uparrow\left(P_{t}\right) \cap p r e^{*}\left(\uparrow P_{t}^{\prime}\right)$. Every configuration $C_{t}$ in $\uparrow\left(P_{t}\right) \cap \operatorname{Pre}^{*}\left(\uparrow P_{t}^{\prime}\right)$ embeds $P_{t}$, and for each of them, there is a tagged run that leads to a configuration $C_{t}^{\prime}$ that embeds $P_{t}^{\prime}$. One can also decide if this run does not meet $T$, using the technique of proposition 11. Furthermore, there can be several agents carrying the same tag in the upward closure of a set of tagged configurations, but for each configuration in $\uparrow\left(P_{t}\right) \cap \operatorname{Pre}^{*}\left(\uparrow P_{t}^{\prime}\right)$, there exists a smaller configuration with only one occurrence of each tag in $T$ and $\perp$ tags everywhere else. As each tag is used only once, there exists a run $\rho_{C, C^{\prime}}^{t}$ starting from $C_{t}$ (with unique use of tags) and leading to $C_{t}^{\prime}$ (with unique use of tags too) such that for every tag $t$, the agent tagged by $t$ in $C_{t}$ and in $C_{t}^{\prime}$ is the same. Hence, if $i$ ) and $\left.i i\right)$ hold, we know that there exists a run $C_{0} \leadsto C \sim C^{\prime}$ where $C=U T\left(C^{t}\right)$ and $C^{\prime}=U T\left(C^{\prime t}\right)$ that matches $\left(P, P^{\prime}, \psi\right)$.

Proof: Lemma 15 just casts a correlated pattern matching problem into two coverability problems, but does not show that they have effective solutions. The proof of this theorem simply consists in showing the effectiveness of Lemma 15 We have already shown that checking whether a run over $\mathcal{C}^{k}$ starting from a configuration $C_{0}$ covers some configuration $C$ is effective for any choice of $C_{0}$. Hence, part $i$ ) of Lemma 15 is effective.

Similarly, checking if there exists a run starting from $C_{0}$ and reaching a configuration in an upward closed set $X$ represented by its basis $\mathcal{B}_{X}=\left\{B_{1}, \ldots B_{n}\right\}$ simply consists in checking coverability from $C_{0}$ for some $B_{i} \in \mathcal{B}_{X}$. Hence, if $\uparrow P_{t} \cap \operatorname{Pre} *\left(\uparrow P_{t}^{\prime}\right)$ can be represented by a finite basis, part $i i$ ) of Lemma 15 is effective. Let us now prove that $T \mathcal{C}^{k}$ is intersection effective, which guarantees that $\uparrow P_{t} \cap \operatorname{Pre}^{*}\left(\uparrow P_{t}^{\prime}\right)$ can be represented by a finite basis. For a pair of tagged configurations $C_{1}, C_{2}$ (with same set of used tags $T$ ), one can define a pair of partial mappings $\gamma: V_{1} \rightarrow V_{2}, \gamma^{\prime}: E_{1} \rightarrow E_{2}$ that respects agents and sessions labels and edges (i.e. $\tau(\gamma(v))=\gamma(v)$ and for every $\left.e=\left(v, l, v^{\prime}\right), \gamma^{\prime}(e)=\left(\gamma(v), l, \gamma\left(v^{\prime}\right)\right)\right)$. Then, the configuration $C_{1,2}^{\gamma, \gamma^{\prime}}=\left(V_{1} \uplus V_{2} \backslash i m g(\gamma), E_{1} \uplus E_{2} \backslash i m g\left(\gamma^{\prime}\right), \tau_{1} \cup \tau_{2}, T\right)$, i.e. the configuration that coalesces similar pairs of vertices in $\gamma$ and edges in $\gamma^{\prime}$ embeds $C_{1}$ and $C_{2}$. That is $C_{1,2}^{\gamma, \gamma^{\prime}}$ belongs to $\uparrow C_{1} \cap \uparrow C_{2}$. For a fixed pair of tagged configurations, there exists only a finite number of such mappings, that we will denote by $\Delta$. We now have to show that the minimal elements of $G_{1 \cap 2}=\left\{C_{1,2}^{\gamma, \gamma^{\prime}} \mid\left(\gamma, \gamma^{\prime}\right)\right.$ is a mapping from $C_{1}$ to $\left.C_{2}\right\}$ form a basis for $\uparrow C_{1} \cap \uparrow C_{2}$. Let $x \in \uparrow C_{1} \cap \uparrow C_{2}$. Then, there exists an embedding $\psi_{1, x}$ from $C_{1}$ to $x$, and another one $\psi_{2, x}$ from $C_{2}$ to $x$, as shown in Figure 6. We can show that $\psi_{1, x} \circ \psi_{2, x}^{-1}$ is a partial mapping $\psi_{x}=\left(\gamma_{x}, \gamma_{x}^{\prime}\right)$ from $C_{1}$ to $C_{2}$ (the image of a vertex in $C_{1}$ by $\psi_{1, x}$ is not always the image of a vertex of $C_{2}$ by $\psi_{2, x}$, and similarly for edges), that belongs to $\Delta$. Hence, the configuration $C_{1,2}^{\gamma_{x}, \gamma_{x}^{\prime}}$ belongs to $G_{1 \cap 2}$, and there exists a pair of embeddings $\psi_{1}$ of $C_{1}$ into $C_{1,2}^{\gamma_{x}, \gamma_{x}^{\prime}}$ and $\psi_{2}$ of $C_{2}$ into $C_{1,2}^{\gamma_{x}, \gamma_{x}^{\prime}}$ (the embeddings due to inclusion $\sqsubseteq_{t}$ ). Then, the mapping $\psi_{12, x}$ that associates vertices in $i m g\left(\psi_{1}\right)$ (resp edges) with their image by $\psi_{1}^{-1} \circ \psi_{1, x}$ and vertices in $i m g\left(\psi_{2}\right) \backslash i m g\left(\psi_{1}\right)$ (resp. edges) with their image by $\psi_{2}^{-1} \circ \psi_{2}, x$ is an embedding from $C_{1,2}^{\gamma_{x}, \gamma_{x}^{\prime}}$ to $x$. Hence, we have $C_{1,2}^{\gamma_{x}, \gamma_{x}^{\prime}} \sqsubseteq x$. This property holds for any $x \in \uparrow C_{1} \cap \uparrow C_{2}$, so $\min \left(G_{1 \cap 2}\right)$ is a basis for $\uparrow C_{1} \cap \uparrow C_{2}$, and $T C^{k}$ is intersection effective.

The basis of $\operatorname{Pre}^{*}\left(\uparrow P_{t}^{\prime}\right)$ is a finite set $\left\{B_{1}, \ldots, B_{n}\right\}$, and for any upward closed set $X$ of configurations, we have $\uparrow X \cap \operatorname{Pre}^{*}\left(\uparrow P_{t}^{\prime}\right)=\uparrow\left\{X \cap \uparrow B_{i}\right\}_{i \in 1 . . n}$, which can be represented by a finite basis. Hence, part $i i)$ of lemma 15 can be effectively checked. Given a Session system $S$, and a corelated pattern $\left(P, P^{\prime}, \psi\right)$, one can decide if there exists a run of $S$ over $\mathcal{C}^{k}$ matching $\left(P, P^{\prime}, \psi\right)$. The result extends to CWPs buy considering one correlated pattern after another.

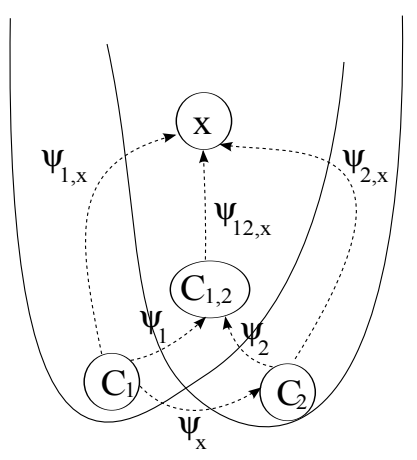

Fig. 6. Intersection effectiveness 